\title{
Coexistence of fast and slow gamma oscillations in one population of inhibitory spiking neurons
}

\author{
Hongjie Bi, ${ }^{1, *}$ Marco Segneri, ${ }^{1, \dagger}$ Matteo di Volo, ${ }^{1,2, \$}$ and Alessandro Torcini ${ }^{1, \S}$ \\ ${ }^{1}$ Laboratoire de Physique Théorique et Modélisation, Université de Cergy-Pontoise, CNRS, UMR No. 8089, \\ 95302 Cergy-Pontoise Cedex, France \\ ${ }^{2}$ Unité de Neuroscience, Information et Complexité, CNRS FRE No. 3693, 1 Avenue de la Terrasse, 91198 Gif sur Yvette, France
}

(Received 28 December 2018; revised manuscript received 29 June 2019; published 13 January 2020)

\begin{abstract}
Oscillations are a hallmark of neural population activity in various brain regions with a spectrum covering a wide range of frequencies. Within this spectrum $\gamma$ oscillations have received particular attention due to their ubiquitous nature and their correlation with higher brain functions. Recently, it has been reported that $\gamma$ oscillations in the hippocampus of behaving rodents are segregated in two distinct frequency bands: slow and fast. These two $\gamma$ rhythms correspond to different states of the network, but their origin has been not yet clarified. Here we show theoretically and numerically that a single inhibitory population can give rise to coexisting slow and fast $\gamma$ rhythms corresponding to collective oscillations of a balanced spiking network. The slow and fast $\gamma$ rhythms are generated via two different mechanisms: the fast one being driven by the coordinated tonic neural firing and the slow one by endogenous fluctuations due to irregular neural activity. We show that almost instantaneous stimulations can switch the collective $\gamma$ oscillations from slow to fast and vice versa. Furthermore, to draw a connection with the experimental observations, we consider the modulation of the $\gamma$ rhythms induced by a slower $(\theta)$ rhythm driving the network dynamics. In this context, depending on the strength of the forcing and the noise amplitude, we observe phase-amplitude and phase-phase coupling between the fast and slow $\gamma$ oscillations and the $\theta$ forcing. Phase-phase coupling reveals on average different $\theta$-phase preferences for the two coexisting $\gamma$ rhythms joined to a wide cycle-to-cycle variability.
\end{abstract}

DOI: 10.1103/PhysRevResearch.2.013042

\section{INTRODUCTION}

The emergence of collective oscillations in complex system has been a subject largely studied in recent decades from an experimental as well as from a theoretical point of view (for a recent review see [1]). In particular, the transition from asynchronous to collective dynamics in networks of heterogeneous oscillators has been characterized in terms of methods borrowed from statistical mechanics [2-4] and nonlinear dynamics [5-7]. Exact analytic techniques to reduce the infinite-dimensional dynamics of globally coupled inhomogeneous phase oscillators to a few mean-field variables have became available in the past decade [8], allowing for noticeable progress in the field [1]. Quite recently, these reduction techniques have been applied to globally coupled spiking neural networks [9], thus opening alternative perspectives for the study of large ensembles of spiking neurons and for the understanding of the mechanisms underlying brain rhythms.

Oscillatory dynamics is fundamental for the functioning of the mammalian brains. Rhythms ranging from 1 to $500 \mathrm{~Hz}$

\footnotetext{
*hongjie.bi@u-cergy.fr

†marco.segneri@u-cergy.fr

†matteo.divolo@unic.cnrs-gif.fr

$\S$ alessandro.torcini@u-cergy.fr
}

Published by the American Physical Society under the terms of the Creative Commons Attribution 4.0 International license. Further distribution of this work must maintain attribution to the author(s) and the published article's title, journal citation, and DOI. have been measured at a mesoscopic level, corresponding to the dynamics of neural populations, by employing electroencephalography, magnetoencephalography, or local field potential (LFP) [10].

In particular, $\gamma$ oscillations $(30-100 \mathrm{~Hz})$ have been suggested to underlie various cognitive and motor functions. Oscillations in the $\gamma$ band have been related to attention selection [11], memory formation and retrieval [12,13], binding mechanisms for sensory awareness [14], and human focal seizures [15].

Gamma oscillations have been observed in many areas of the brain and their emergence has been shown to be crucially dependent on inhibitory networks $[16,17]$. It was shown in [16] that $\gamma$ oscillations in purely inhibitory networks can emerge only via two mechanisms: Single neurons can fire periodically locked in phase [18] or each neuron can have irregular activity, but sufficiently strong recurrent interactions can render the asynchronous state unstable against fluctuations and collective oscillations (COs) can arise [19-21]. The role of the synaptic mechanisms in promoting tonic synchronization in the $\gamma$ range has been clarified in [17,22], while [23-27] have shown that fast network oscillations with irregular neural discharges can emerge when the neurons are operating in the so-called balanced state. This is a typical cortical state where the balance of excitation and inhibition allows for healthy activity in the brain. The balanced state has been observed in vitro and in vivo experiments in the cerebral cortex [28,29] and reported in simulations of networks of excitatory and inhibitory spiking neurons $[20,30,31]$ as well as of purely inhibitory circuits driven by external excitatory currents [32,33]. 
Gamma oscillations are usually modulated by $\theta$ oscillations in the hippocampus during locomotory actions and rapid eye movement (REM) sleep; $\theta$ frequencies correspond to $4-12 \mathrm{~Hz}$ in rodents $[34,35]$ and to $1-4 \mathrm{~Hz}$ in humans $[36,37]$. Two mechanisms of entrainment (or cross-frequency coupling) between $\theta$ and $\gamma$ oscillations have been reported, namely, phase-amplitude (PA) and phase-phase (PP) coupling. The PA coupling (or $\theta$-nested $\gamma$ oscillations) corresponds to the fact that the phase of the $\theta$ oscillation modifies the amplitude of the $\gamma$ waves [38,39], while PP coupling refers to $n: m$ phase locking between $\gamma$ - and $\theta$-phase oscillations $[35,40]$.

Recently, the coexistence of $\gamma$ oscillations in three distinct bands has been reported for the cornu ammonis area 1 (CA1) of the hippocampus [35], namely, a slow one $(\simeq 30-50 \mathrm{~Hz})$, a fast (or intermediate) one $(\simeq 50-90 \mathrm{~Hz}$ ), and a so-called $\varepsilon$ band ( $\simeq 90-150 \mathrm{~Hz}$ ). However, only the two lower bands show a clear correlation (PP coupling) with the $\theta$ rhythm during maze exploration and REM sleep, thus suggesting their functional relevance [35]. There is further evidence that these two $\gamma$ bands correspond to different states of the hippocampal network [41]. In particular, in freely behaving rats, place cells code differently the space location and the running speed during $\theta$-nested slow or fast $\gamma$ rhythms [41-43]. Moreover, $\gamma$ rhythms with similar low- and high-frequency subtypes occur in many other brain regions besides the hippocampus $[44,45]$. Despite their relevance, the mechanisms behind the emergence of these two distinct $\gamma$ bands are not yet clarified.

Concerning the hippocampus, experiments show that slow $\gamma$ rhythms couple the activity of the CA1 to synaptic inputs from CA3, while fast $\gamma$ rhythms in CA1 are entrained by inputs from medial entorhinal cortex (mEC) [45]. Slow and fast oscillations have been recorded also in CA3, where fast $\gamma$ are entrained by synaptic inputs from mEC [46]. These findings suggest that CA3-activated interneurons drive slow $\gamma$, while mEC-activated interneurons drive fast $\gamma$. Nonetheless, it has been shown that a substantial proportion of CA1 interneurons phase lock to both slow and fast $\gamma$ LFP oscillations $[35,46,47]$. Therefore, as suggested by Colgin in [45], such interneurons may be part of a network that can generate either slow or fast $\gamma$, depending on the state of the network. Furthermore, there is experimental evidence that $\gamma$ rhythms can be generated locally in vitro in the CA1, as well as in the CA3 and mEC, due to optogenetic stimulations $[39,48,49]$ or pharmacological manipulations, but at lower $\gamma$ frequencies with respect to optogenetics [50-53]. A recent theoretical work has analyzed the emergence of $\gamma$ oscillations in a neural circuit composed of two populations of interneurons with fast and slow synaptic timescales [54]. Based on the results of this idealized rate model and on the analysis of experimental data sets for the CA1, the authors showed that multiple $\gamma$ bands can arise locally without being the reflection of feedforward inputs.

In the present work we show that a single inhibitory population, characterized by only one synaptic time, can display coexisting fast and slow $\gamma$ COs corresponding to different network states. In particular, the slow $\gamma$ oscillations are associated with irregular spiking behaviors and fluctuations driven, while the fast $\gamma$ oscillations coexist with a much more regular neural dynamics and can be characterized as mean driven [55,56]. Furthermore, in the presence of $\theta$ forcing, we observe different $\theta-\gamma$ cross-frequency coupling scenarios depending on the forcing amplitude. For small amplitudes we have $\theta$-nested $\gamma$ oscillations resembling those reported for various brain areas in vitro under optogenetic sinusoidal $\theta$ stimulation $[39,48,49]$. At larger amplitudes the two types of $\gamma$ COs phase lock to the $\theta$ rhythm, similarly to what has been reported experimentally for the CA1 region of the hippocampus [35,46]. More specifically, we have studied balanced sparse inhibitory networks of quadratic integrateand-fire (QIF) neurons pulse coupled via inhibitory postsynaptic potentials (IPSPs), characterized by a finite synaptic timescale. For this sparse network we derived an effective mean field by employing recently developed reduction techniques for QIF networks $[9,21,57,58]$. In the mean-field (MF) model, in proximity to the subcritical Hopf bifurcations, we report regions of bistability involving one stable focus and one stable limit cycle. In direct simulations of the corresponding spiking network we observe the coexistence of two distinct COs with frequencies in the slow and fast $\gamma$ bands. The slow $\gamma$ COs are due to the microscopic irregular dynamics, characteristic of the balanced dynamics, which turns the damped oscillations towards the MF focus in sustained COs. The fast $\gamma$ COs are instead related to the oscillatory branch emerging via the subcritical Hopf bifurcation from the asynchronous state. The network can be driven from one kind of $\mathrm{CO}$ to the other by transiently stimulating the neurons. In the presence of a $\theta$ forcing, nested $\gamma$ oscillations characterized by a PA coupling appear for small forcing amplitudes, while at intermediate amplitudes slow and fast $\gamma$ phases lock to the $\theta$ phase displaying PP coupling between the rhythms. For even larger amplitudes only fast $\gamma$ are observable, with a maximal power corresponding to the maximum of the stimulation.

The paper is organized as follows. In Sec. II we introduce the model for an inhibitory sparsely balanced network of QIF neurons as well as the macroscopic and microscopic indicators employed to characterize its dynamics. Section III is devoted to the derivation of the corresponding effective MF model and to the linear stability analysis of the asynchronous state. Simulation results for the network for high and low structural heterogeneity are reported in Sec. IV and compared with MF forecasts. The coexistence and transitions from slow (fast) to fast (slow) $\gamma$ oscillations are analyzed in Sec. V together with the cross-frequency coupling between $\theta$ and $\gamma$ oscillations. A concise discussion of the results and possible future developments is reported in Sec. VI. Appendix A is devoted to the analysis of coexisting $\gamma$ oscillations in ErdősRényi networks and Appendix B discusses a general mechanism for the coexistence of noise-driven and tonic oscillations.

\section{METHODS}

\section{A. Network model}

We consider $N$ inhibitory pulse-coupled QIF neurons [59] arranged in a random sparse balanced network. The membrane potential of each neuron evolves according to

$$
\begin{aligned}
& \tau_{m} \dot{v}_{i}(t)=I+v_{i}^{2}(t)-\tau_{m} J y_{i}(t), \\
& \tau_{d} \dot{y}_{i}(t)=-y_{i}(t)+\sum_{j} \epsilon_{j i} \delta\left(t-t_{j}(m)\right),
\end{aligned}
$$


where $\tau_{m}=15 \mathrm{~ms}$ represents the membrane time constant and $I$ an external DC, encompassing the effects of distal excitatory inputs and of the internal neural excitability. The last term in (1a) is the inhibitory synaptic current, with $J$ the synaptic coupling and $y_{i}$ the synaptic field experienced by neuron $i$. Whenever the membrane potential $v_{i}$ reaches infinity, a spike is emitted and $v_{i}$ is reset to $-\infty$.

The field $y_{i}$ is the linear superposition of all the exponential IPSPs $s(t)=\exp \left(-t / \tau_{d}\right)$ received by the neuron $i$ from its presynaptic neurons in the past, namely,

$$
y_{i}(t)=\frac{1}{\tau_{d}} \sum_{j \in \operatorname{pre}(i)} \sum_{m \mid t_{j}(m)<t} \epsilon_{j i} \Theta\left(t-t_{j}(m)\right) s\left(t-t_{j}(m)\right),
$$

where $\tau_{d}$ is the synaptic time constant, $t_{j}(m)$ is the spike time of the $m$ th spike delivered by the $j$ th neuron, $\Theta(t)$ is the Heaviside function, and $\epsilon_{j i}$ is the adjacency matrix of the network. In particular, $\epsilon_{j i}=1(0)$ if a connection from node $j$ to $i$ exists (or not) and $k_{i}=\sum_{j} \epsilon_{j i}$ is the number of presynaptic neurons connected to neuron $i$, or in other terms its in-degree.

In order to compare the simulation results with an exact mean field derived recently $[9,21,57]$, we consider sparse networks where the in-degrees $k_{i}$ are extracted from a Lorentzian distribution

$$
P(k)=\frac{\Delta_{k}}{(k-K)^{2}+\Delta_{k}^{2}}
$$

peaked at $K$ and with a half-width at half maximum (HWHM) $\Delta_{k}$, the parameter $\Delta_{k}$ measures the level of structural heterogeneity in the network, and analogously to Erdős-Rényi networks we assume the following scaling for the HWHM: $\Delta_{k}=\Delta_{0} \sqrt{K}$. The DC and the synaptic coupling are rescaled with the median in-degree $K$ as $I=I_{0} \sqrt{K}$ and $J=J_{0} / \sqrt{K}$, as usually done to achieve a self-sustained balanced state for sufficiently large in-degrees [23-25,27,31]. In this paper we will usually consider $I_{0}=0.25, N=10000$, and $K=1000$, unless stated otherwise.

\section{B. Simulation protocols}

The network dynamics is integrated by employing a standard Euler scheme with an integration time step $\Delta t=$ $\tau_{m} / 10000$. The coexistence of solutions in proximity to a subcritical Hopf bifurcation is analyzed by performing adiabatic network simulations where a control parameter, e.g., the synaptic time $\tau_{d}$, is slowly varied. In particular, these are performed by starting with an initial value of $\tau_{d}^{(0)}$ and arriving at a final value $\tau_{d}^{(1)}$ in $M$ steps, each time increasing $\tau_{d}$ by $\Delta \tau_{d}=\left(\tau_{d}^{(1)}-\tau_{d}^{(0)}\right) /(M-1)$. Once the final value $\tau_{d}^{(1)}$ is reached, the synaptic time is decreased in steps $\Delta \tau_{d}$ down to $\tau_{d}^{(0)}$. Each step corresponds to a simulation for a time $T_{s}=90 \mathrm{~s}$ during which the quantities of interest are measured, after discarding a transient $T_{t}=15 \mathrm{~s}$. The initial condition for the system at each step is its final configuration at the previous step.

Concerning the analysis of the crossing times $t_{c}$ from slow (fast) to fast (slow) $\gamma$ in a bistable regime, reported in Sec. VA, we proceed as follows. Let us first consider the transition from slow to fast $\gamma$ COs. We initialize the system in the slow $\gamma$ state at a current $I_{0} \equiv I_{1}$ ensuring the bistability of the dynamics. Then we increase the DC to a value $I_{0} \equiv I_{2}$ for a time interval $T_{P}$, after which we return to the original value $I_{0} \equiv I_{1}$ and we check, after a period of $1.5 \mathrm{~s}$, if the system is in the slow or fast $\gamma$ regime. Then we repeat the process $M=30$ times for each value of $T_{P}$ considered and we measure the corresponding transition probability. The crossing time $t_{c}$ is defined as the minimal $T_{P}$ giving $80 \%$ probability that the transition will take place. To analyze the transition from fast to slow, we initialize the system in the fast $\gamma$ state at a DC $I_{1}$, decrease the current to a value $I_{0} \equiv I_{3}$ for time $T_{P}$, and then proceed as before. To examine the influence of noise on such transitions we add to the membrane potential evolution a noise term of zero average and amplitude $A_{n}$.

\section{Indicators}

To characterize the collective dynamics in the network we measure the mean membrane potential $V(t)=\sum_{i=1}^{N} v_{i}(t) / N$, the instantaneous firing rate $R(t)$, corresponding to the number of spikes emitted per unit of time and per neuron, and the mean synaptic field $Y(t)=\sum_{i=1}^{N} y_{i}(t) / N K{ }^{1}$

The microscopic activity can be analyzed by considering the interspike interval (ISI) distribution as characterized by the coefficient of variation $\mathrm{cv}_{i}$ for each neuron $i$, which is the ratio between the standard deviation and the mean of the ISIs associated with the train of spikes emitted by the considered neuron. In particular, we will characterize each network in terms of the average coefficient of variation defined as $\mathrm{CV}=\sum_{i} \mathrm{cv}_{i} / N$. Time averages and fluctuations are usually estimated on time intervals $T_{s} \simeq 90 \mathrm{~s}$, after discarding transients $T_{t} \simeq 15 \mathrm{~s}$.

Phase entrainment between an external forcing characterized by its phase $\theta(t)$ and the collective oscillations induced in the network can be examined by considering the phase difference

$$
\Delta_{n m}(t)=n \theta(t)-m \gamma(t),
$$

where $\gamma(t)$ is the phase of the COs defined by considering the time occurrences $T_{k}$ of the $k$ maximum of the instantaneous firing rate $R(t)$ of the network, namely, $\gamma(t)=2 \pi(t-$ $\left.T_{k}\right) /\left(T_{k+1}-T_{k}\right)$ with $t \in\left[T_{k}, T_{k+1}\right] .^{2}$ We have an $n: m$ phase locking whenever the phase difference (4) is bounded during the time evolution, i.e., $\left|\Delta_{n m}(t)\right|<$ const.

This somehow qualitative criterion can be made more quantitative by considering statistical indicators measuring the level of $n: m$ synchronization for irregular or noisy data. In particular, an indicator based on the Shannon entropy has been introduced in [40], namely,

$$
e_{n m}=\frac{E_{\max }-E}{E_{\max }} \quad \text { with } E=-\sum_{k=1}^{M} p_{k} \ln \left(p_{k}\right),
$$

\footnotetext{
${ }^{1}$ In the definition of the mean synaptic field we have divided the sum also by the median in-degree $K$ because on average a neuron is subject to $K$ spike trains.

${ }^{2}$ This definition of the phase avoids spurious phase-locking indications in terms of the Kuramoto order parameter arising with imperfectly harmonic signals as pointed out in [61].
} 
where $E$ is the entropy associated with the distribution of $\Delta_{n m}(t)$ and $E_{\max }=\ln (M)$ with $M$ number of bins.

The degree of synchronization among the phases can be also measured by the so-called Kuramoto order parameter, namely $[35,60]$,

$$
\rho_{n m}=\left|\frac{1}{L} \sum_{k=1}^{L} e^{i \Delta_{n m}\left(t_{k}\right)}\right|,
$$

where $|\cdot|$ represents the modulus and $t_{k}=k \frac{T_{W}}{L}$ are $L$ successive equispaced times within the considered time window $T_{W}$. For completely desynchronized phases $\rho_{n m} \propto 1 / \sqrt{L}$, while partial (full) synchronization will be observable whenever $\rho_{n m}$ is finite (one).

To assess the stationarity and the statistical significance of the obtained data we measured the above indicators within a time window $T_{W}$ and we averaged the results over several distinct time windows in order to obtain also the corresponding error bars. Furthermore, to avoid the detection of spurious phase locking due to noise or bandpass filtering one should derive significance levels $e_{n m}^{(S)}$ and $\rho_{n m}^{(S)}$ for each $n: m$ phase locking indicators $e_{n m}$ and $\rho_{n m}$ [40,61]. The significance levels have been estimated by considering surrogate data obtained by randomly shuffling the original time stamps of one of the two phases considered. Moreover, following [61], we considered also two other types of surrogates for the generation of $\Delta_{n m}(t)$ (4) within a certain time window $T_{W}$ : the time-shift surrogate, obtained by time shifting the origin of one time series for the phases with respect to the original one in the definition of (4), and the random permutation surrogate, obtained by randomly choosing the origins of two time windows of duration $T_{W}$ to estimate $\Delta_{n m}(t)$.

\section{EFFECTIVE MEAN-FIELD MODEL FOR A SPARSE QIF NETWORK}

Following [21], we derive an effective MF formulation for the model (1). As a starting point we consider an exact macroscopic model recently derived for fully coupled networks of pulse-coupled QIF neurons [9]; in particular we focus on inhibitory neurons coupled via exponentially decaying IPSPs [57]. For a structurally inhomogeneous network made of identical QIF neurons, with the synaptic couplings randomly distributed according to a Lorentzian, the MF dynamics can be expressed in terms of only three collective variables (namely, $V, R$, and $Y$ ), as

$$
\begin{aligned}
\tau_{m} \dot{R} & =2 R V+\frac{\Gamma}{\pi} Y, \\
\tau_{m} \dot{V} & =V^{2}+I+\bar{g} \tau_{m} Y-\left(\pi \tau_{m} R\right)^{2}, \\
\tau_{d} \dot{Y} & =-Y+R,
\end{aligned}
$$

where $\bar{g}$ is the median and $\Gamma$ the HWHM of the Lorentzian distribution of the synaptic couplings.

At a mean-field level, the above formulation can be applied to a sparse network; indeed, the quenched disorder in the connectivity distribution can be rephrased in terms of a random synaptic coupling. Namely, each neuron $i$ is subject on average to an inhibitory synaptic current of amplitude $g_{0} k_{i} Y / \sqrt{K}$ proportional to its in-degree $k_{i}$. Therefore, at a first (a)

(b)
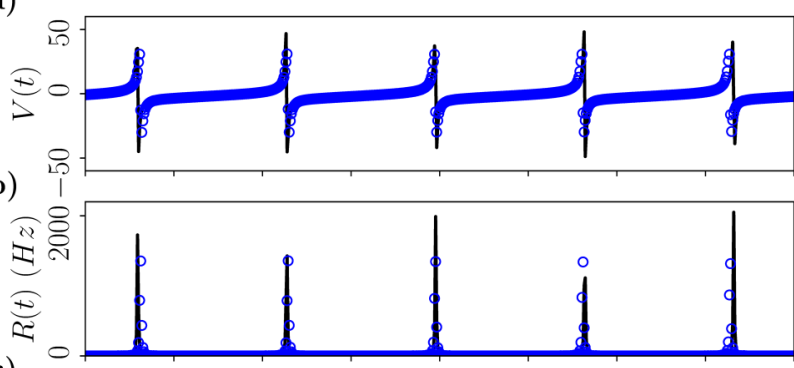

(c)

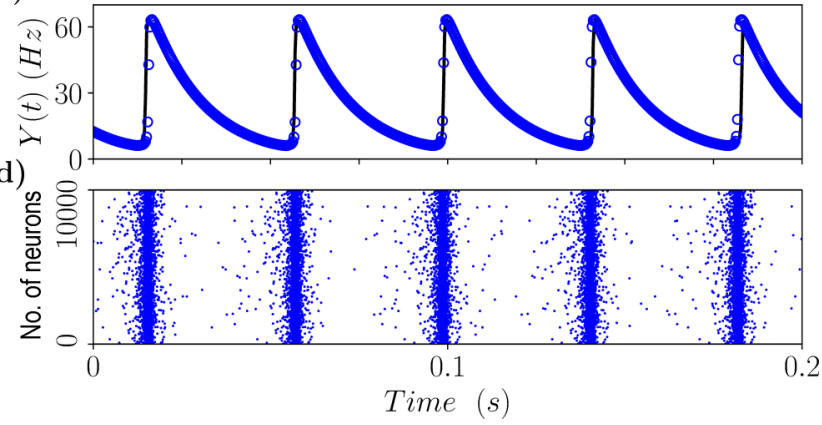

FIG. 1. Comparison of the spiking dynamics with the mean-field results. Collective variables (a) $V$, (b) $R$, and (c) $Y$ versus time, obtained from simulations of the spiking network (1) (blue circles) and from the MF formulation (8) (black line). (d) Corresponding raster plot, revealing clear COs with frequency $v_{\text {osc }} \simeq 24 \mathrm{~Hz}$. The dynamics of the network is for $N=10000$ neurons with median indegree $K=1000$ and $\Delta_{0}=0.3$. The other parameters are $I_{0}=0.25$, $J_{0}=1.0$, and $\tau_{d}=15 \mathrm{~ms}$.

level of approximation we can consider the neurons as fully coupled but with random values of the coupling distributed as a Lorentzian of median $\bar{g}=-J_{0} \sqrt{K}$ and HWHM $\Gamma=J_{0} \Delta_{0}$. The MF formulation (7) is now expressed as

$$
\begin{aligned}
\tau_{m} \dot{R} & =2 R V+\frac{\Delta_{0} J_{0}}{\pi} Y, \\
\tau_{m} \dot{V} & =V^{2}+\sqrt{K}\left(I_{0}-J_{0} \tau_{m} Y\right)-\left(\pi \tau_{m} R\right)^{2}, \\
\tau_{d} \dot{Y} & =-Y+R .
\end{aligned}
$$

As verified in [21], for instantaneous PSPs this formulation represents a quite good guidance for the understanding of the emergence of sustained COs in the network, despite the fact that the MF asymptotic solutions are always stable foci. Instead, in the present case, analogously to what was found for structurally homogeneous networks of heterogeneous neurons in [57], we observe that for IPSPs of finite duration oscillations can emerge in the network as well as in the mean field, as shown in Fig. 1. The data reported in the figure confirm that the MF formulation (8), despite not including current fluctuations, reproduces quite well the macroscopic evolution of the network in the oscillatory regime also for a sparse network. Therefore, we can safely employ such effective MF model to interpret the phenomena observed in the spiking network and to obtain theoretical predictions for its dynamics.

In the two following sections we will first study analytically the linear stability of the asynchronous state, which corresponds to a fixed point of (8), and then describe the 
bifurcation and phase diagrams associated with the MF model (8).

\section{A. Linear stability of the asynchronous state}

The fixed point solution $\left(V^{*}, R^{*}, Y^{*}\right)$ of $(8)$ is given by

$$
\begin{aligned}
V^{*} & =-\frac{\Delta_{0} J_{0}}{2 \pi}, \\
R^{*} \tau_{m} & =\frac{J_{0} \sqrt{K}}{2 \pi^{2}}\left(\sqrt{1+\frac{4 \pi^{2}}{\sqrt{K}} \frac{I_{0}}{J_{0}^{2}}+\frac{\Delta_{0}^{2}}{K}}-1\right), \\
Y^{*} & =R^{*} .
\end{aligned}
$$

By performing a linear stability analysis around the fixed point solution $\left(V^{*}, R^{*}, Y^{*}\right)$ we obtain the secular equation

$$
\left|\begin{array}{ccc}
2 V^{*}-\Lambda \tau_{m} & 2 R^{*} & -2 V^{*} \\
-2\left(\pi \tau_{m}\right)^{2} R^{*} & 2 V^{*}-\Lambda \tau_{m} & -J_{0} \sqrt{K} \tau_{m} \\
1 & 0 & -1-\Lambda \tau_{d}
\end{array}\right|=0 .
$$

In a more explicit form this is

$$
\begin{aligned}
& \left(1+\Lambda \tau_{d}\right)\left[\left(\Lambda \tau_{m}-2 V^{*}\right)^{2}+\left(2 \pi R^{*} \tau_{m}\right)^{2}\right] \\
& \quad+2 V^{*}\left(\Lambda \tau_{m}-2 V^{*}\right)+2 J_{0} \sqrt{K} R^{*} \tau_{m}=0 .
\end{aligned}
$$

In the present case, for inhibitory coupling, i.e., $J_{0}>0$, the solutions of the cubic equation (11) are one real and two complex conjugates. The real one is always negative (thus irrelevant for the stability analysis), while the couple of complex eigenvalues $\Lambda=\Lambda_{R} \pm i \Lambda_{I}$ can cross the imaginary axes, giving rise to oscillatory behaviors via Hopf bifurcations. The presence of the two complex conjugate eigenvalues implies that whenever the asynchronous state is stable, this is always a focus characterized by a frequency of relaxation towards the fixed point given by $v_{D}=\Lambda_{I} / 2 \pi$. For excitatory coupling, the real eigenvalue can become positive, with an associated saddle-node bifurcation and the emergence of collective chaos $[62,63]$. By following [57], the Hopf boundaries can be identified by setting $\Lambda=i 2 \pi v_{O}$ in (11) and setting equal to zero the real and imaginary parts of the resulting equation, namely, one gets

$$
\begin{gathered}
\frac{\left(1-4 \tau_{d} V^{*}\right)\left(2 \pi v_{O}\right)^{2}}{R^{*}}-(2 \pi)^{2} R^{*} \tau_{m}-2 J_{0} \sqrt{K}=0, \\
{\left[\left(2 \pi v_{O}\right)^{2} \tau_{m}-4\left(V^{*}\right)^{2}-\left(2 \pi R^{*} \tau_{m}\right)^{2}\right]-2 \frac{\tau_{m} V^{*}}{\tau_{d}}=0 .}
\end{gathered}
$$

\section{B. Phase diagrams of the mean-field model}

Apart from the linear stability of the asynchronous state and the associated Hopf boundaries which can be worked out analytically, the limit cycle solutions of the MF model and the associated bifurcations have been obtained by employing the software XPP AUTO developed for orbit continuation [64]. The MF model (8), apart from the membrane time constant $\tau_{m}$, which sets the system timescale, and the median in-degree $K$, which we set equal to 1000 , is controlled by four independent parameters $\Delta_{0}, J_{0}, I_{0}$, and $\tau_{d}$. In the following we will give an overview of the possible behaviors of the MF model in terms of two-parameter phase diagrams for the most relevant combinations of the four parameters mentioned. The results of these analyses are summarized in Figs. 2 and 3.
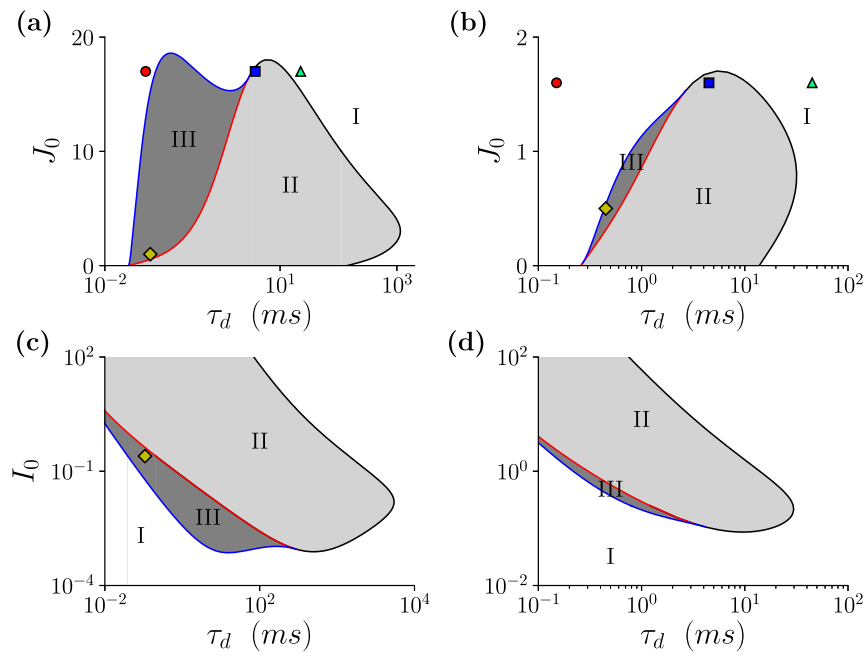

FIG. 2. Phase diagrams of the mean-field model in (a) and (b) the $\left(\tau_{d}, J_{0}\right)$ plane and (c) and (d) the $\left(\tau_{d}, I_{0}\right)$ plane for (a) and (c) $\Delta_{0}=$ 0.3 and (b) and (d) $\Delta_{0}=3$. The red (black) line corresponds to subcritical (supercritical) Hopf bifurcations and the blue line indicates saddle-node bifurcations of limit cycles. In region I (white) the only stable solutions are foci and in region II (lightly shaded) these are limit cycles. The dark shaded area (III) represents the region of coexistence of stable foci and limit cycles. The colored symbols indicate the states analyzed in Sec. IV. The parameters are $K=1000$ and (a) and (b) $I_{0}=0.25$ and (c) and (d) $J_{0}=1.0$.

Our analysis of the stationary solutions has revealed three possible regimes: stable foci (region I), stable COs (region II), and the coexistence of these two stable solutions (region III). The stability boundaries of the COs are delimited by three kind of bifurcations: supercritical Hopf bifurcations (black lines in the figures), subcritical Hopf bifurcations (red lines), and saddle-node bifurcations of limit cycles (blue lines). Stable (unstable) COs emerge from stable foci at supercritical (subcritical) Hopf bifurcations, while stable and unstable limit cycles merge at the saddle-node bifurcations.

A fundamental parameter controlling the emergence of COs in the MF model is the synaptic time $\tau_{d}$; indeed, in the absence of this timescale, no oscillations are present at the MF level [21]. On the other hand, too large values of $\tau_{d}$ also lead to COs suppression, since the present model reduces to a WilsonCowan model for a single inhibitory population, which is know to be unable to display oscillations [57]. As shown in Figs. 2 and 3, oscillations are observable for intermediate values of $\tau_{d}$ and not too large $J_{0}$, since large inhibition leads to a quite reduced activity of the neurons not sufficient to ignite a collective behavior. This is in agreement with the fundamental role played by $\gamma$ aminobutyric acid (GABA) in the emergence of epileptic seizures, characterized by an anomalous level of synchronization among the neurons. Indeed, the occurrence of seizures seems strongly correlated with a GABA deficit, corresponding to a reduction of $J_{0}$ in our case $[65,66]$. Moreover, in order to observe COs, the excitatory drive $I_{0}$ should be larger than some critical value, as shown in Figs. 2(c) and 2(d). This is consistent with the observation of the emergence of $\gamma$ oscillations in hippocampal slices induced through the acetylcholine agonist carbachol [50,67], which leads to a 

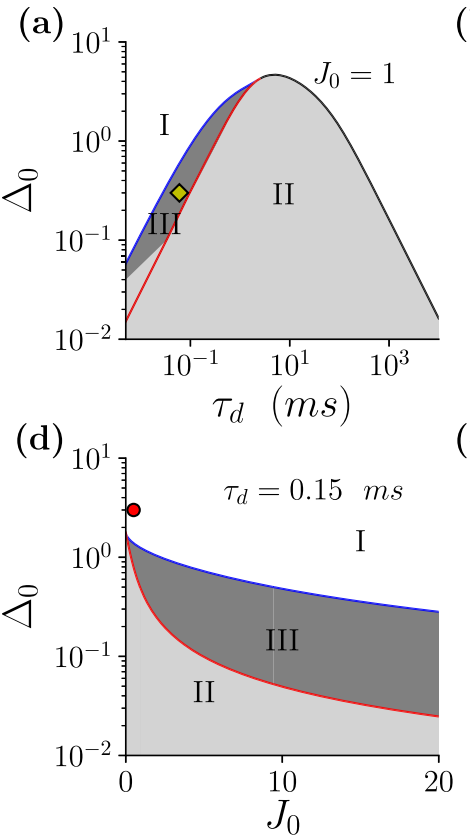

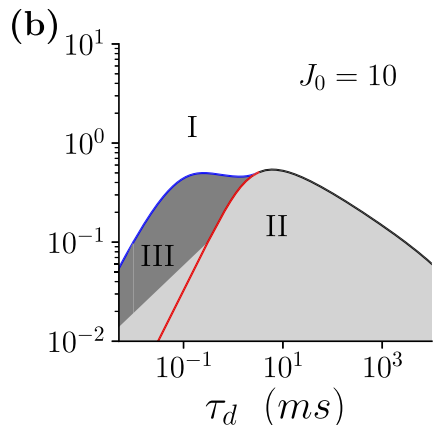

(e)

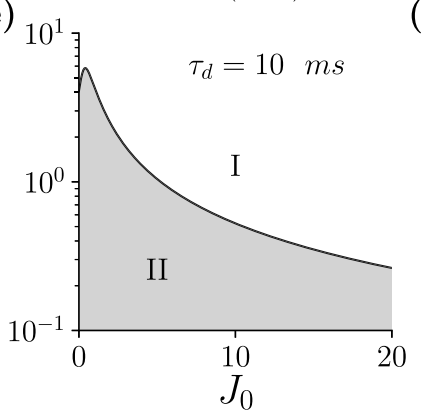

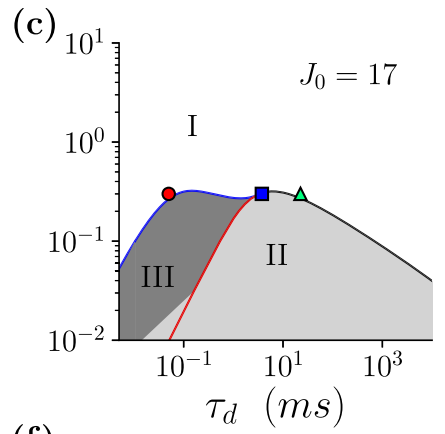

(f)

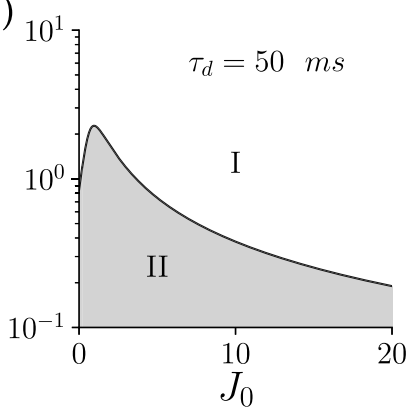

FIG. 3. Phase diagrams of the mean-field model in (a)-(c) the $\left(\tau_{d}, \Delta_{0}\right)$ plane and (d)-(f) the $\left(J_{0}, \Delta_{0}\right)$ plane. The line colors, colored symbols, and regions are defined as in Fig. 2. The parameters are $I_{0}=0.25$ and $K=1000$.

decrease of the conductance of potassium channels, which can be mimicked as an increase of $I_{0}[68,69]$. Indeed, by increasing the structural heterogeneity (measured by $\Delta_{0}$ ), which acts against coherent dynamics, larger values of $I_{0}$ are required for COs as well as smaller synaptic couplings [see Figs. 2(b), 2(d), and 3(d)-3(f)]. Therefore, the emergence of COs can be triggered by self-disinhibition as well as by an external excitatory drive, and we expect to observe in both cases the same scenarios.

As already mentioned, for infinitely fast synapses $\left(\tau_{d} \rightarrow 0\right)$ the only possible solutions of the mean field are foci characterized by two complex conjugate eigenvalues. Nevertheless, in the corresponding network the irregular firings of the neurons, due to the dynamical balance, can sustain COs, which are predicted to relaxed toward the fixed point in the mean field. In the next section we will analyze the role of these microscopic fluctuations in triggering the network dynamics also for finite $\tau_{d}$.

\section{NETWORK DYNAMICS}

We investigate in this section the dynamics of the network by considering the parameter plan $\left(\tau_{d}, J_{0}\right)$. In particular, we want to examine the role of structural heterogeneity (measured by $\Delta_{0}$ ) in shaping the dynamical behaviors. This characteristic of the network structure is extremely relevant, as it can even determine if the system is in a balanced or an imbalanced regime $[21,70,71]$.

\section{A. High structural heterogeneity}

We consider first a relatively high value for the structural heterogeneity, namely, $\Delta_{0}=3.0$. For sufficiently large coupling $J_{0}$, the bifurcation diagram reveals the emergence of oscillations in the MF model (8) via supercritical Hopf bifurcations, analogously to what has been reported for globally coupled networks [57]. An example of the bifurcation diagram, displaying the extrema of the mean membrane potential $V$ as a function of $\tau_{d}$, is reported in Fig. 4(a) for $J_{0}=1.6$. In particular, we observe for instantaneous synapses $\left(\tau_{d} \rightarrow 0\right)$ a stable focus, as expected from the analysis previously reported in [21]. The focus is stable up to $\tau_{1}^{(H)}$, where it is substituted by a stable oscillatory state via a supercritical Hopf bifurcation. Oscillations are observable up to $\tau_{2}^{(H)}$, where via a second supercritical Hopf bifurcation they disappear and the unique stable solution for the MF system remains a focus. The typical stable regimes are denoted in Fig. 4(a) by three capital letters: $A$ corresponds to a focus, $B$ to a limit cycle, and $C$ to another focus. The network dynamics corresponding to these typical MF solutions is examined in the remaining panels of Fig. 4. For the focus solutions the network dynamics is asynchronous, as clearly visible from the corresponding raster plots in Figs. 4(b) and 4(d). Furthermore, the dynamics of the neurons is quite regular in this case, as verified by the values of the average coefficients of variation, namely, $\mathrm{CV} \simeq 0.14$ and $\mathrm{CV} \simeq 0.04$, corresponding to the distributions reported in Figs. 4(e) and 4(f), respectively. At intermediate values of $\tau_{d}$, as predicted by the MF analysis, we observe COs with frequency $v_{\text {osc }} \simeq 34 \mathrm{~Hz}$ in the network dynamics [see Fig. 4(c)]. However, also in this case the dynamics is dominated by suprathreshold neurons with an associated very low $\mathrm{CV}$, as evident from the large peak present at $\mathrm{cv}_{i} \simeq 0$ in the distribution $P\left(\mathrm{cv}_{i}\right)$ shown in Fig. $4(\mathrm{~g})$.

For lower synaptic coupling $J_{0}$ the phase portrait changes, as shown in Fig. 5(a) for $J_{0}=0.5$. In this case the MF analysis indicates that the transition from a stable focus to the oscillatory state occurs by increasing $\tau_{d}$ via a subcritical Hopf bifurcation. At large synaptic coupling, the stable focus is recovered via a supercritical Hopf bifurcation taking place at $\tau_{2}^{(H)}$, analogously to what has been seen for larger coupling. 

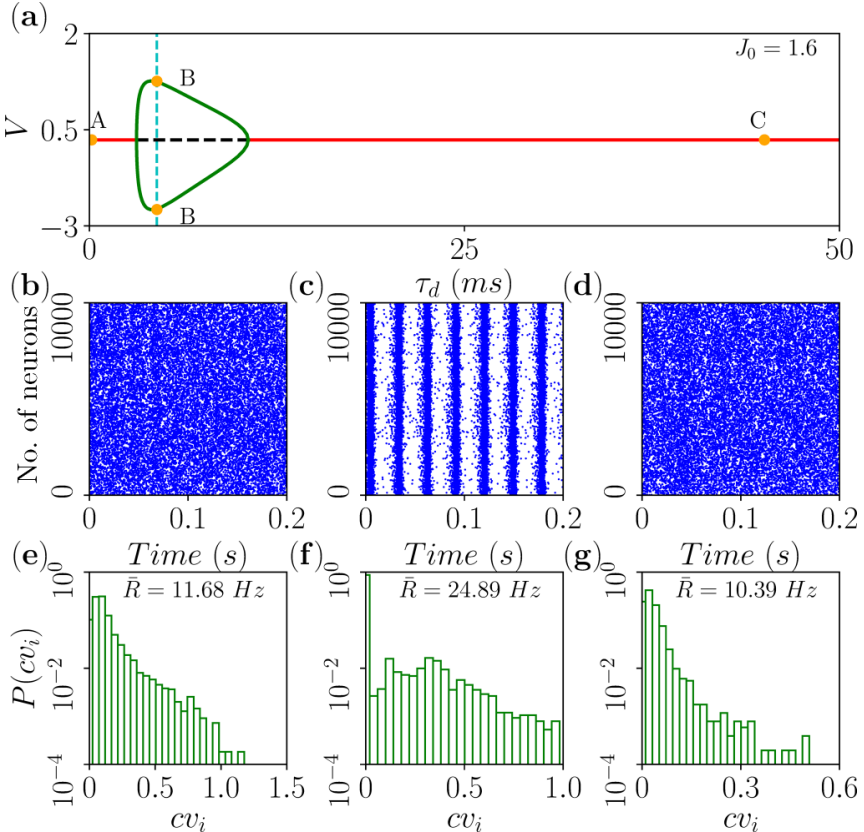

FIG. 4. High structural heterogeneity: supercritical Hopf bifurcation. (a) Bifurcation diagram of the MF model (8) displaying the extrema of $V$ versus $\tau_{d}$. The red solid (black dashed) lines refer to the stable (unstable) focus, while green solid lines refer to the oscillatory state. The supercritical Hopf bifurcations take place for $\tau_{1}^{(H)}=3.14 \mathrm{~ms}$ and $\tau_{2}^{(H)}=10.59 \mathrm{~ms}$. The capital letters in (a) denote three stationary states corresponding to different synaptic timescales, namely, $A, \tau_{d}=0.15 \mathrm{~ms} ; B, \tau_{d}=4.5 \mathrm{~ms}$; and $C, \tau_{d}=45 \mathrm{~ms}$. The network dynamics corresponding to these states is reported in (b) and (e) $A$, (c) and (f) $B$, and (d) and (g) $C$. Also shown are (b)-(d) the corresponding raster plots and (e)-(g) the distributions of the $\left\{\mathrm{cv}_{i}\right\}$ of the single neurons. The network parameters are $N=10000$, $K=1000$, and $\Delta_{0}=3.0$. The other parameters are $I_{0}=0.25$ and $J_{0}=1.6$. The states $A, B$, and $C$ are denoted in Fig. 2(b) as a red circle, a blue square, and a green triangle, respectively.

An interesting regime is observable between $\tau^{(S)}$, where the stable and unstable limit cycles merge via a saddle-node bifurcation, and $\tau_{1}^{(H)}$, where the focus become unstable. In this interval the MF model displays two coexisting stable solutions: a limit cycle and a focus. It is important to verify if also the finite-size sparse network displays this coexistence. Indeed, as shown in Fig. 5, depending on the initial conditions, the network dynamics can converge towards COs or towards an asynchronous state. In particular, we observe that the asynchronous dynamics is associated with extremely low cv values [see Fig. 5(d)], suggesting that this can be considered as a sort of irregular splay state [72]. However, also the COs with $v_{\mathrm{osc}} \simeq 58 \mathrm{~Hz}$ are characterized by a low average coefficient of variation, namely, $\mathrm{CV} \simeq 0.014$, indicating that the dynamics is mean driven. The subcritical Hopf, as expected, is associated with a hysteretic behavior; this effect can be revealed by considering simulations concerning an adiabatic variation of $\tau_{d}$. The results of these simulations are shown in Fig. 5(b), where the maximal values of the instantaneous firing rate $R_{M}$ are given as a function of $\tau_{d}$ for the adiabatic protocol and compared with the MF estimations of $R_{M}$. From the figure it is clear that the transition from the focus to the stable limit cycle (a)

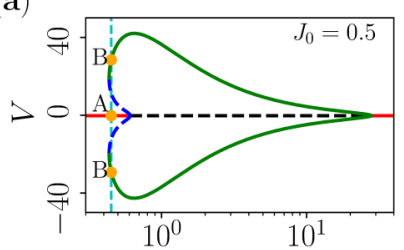

(c)
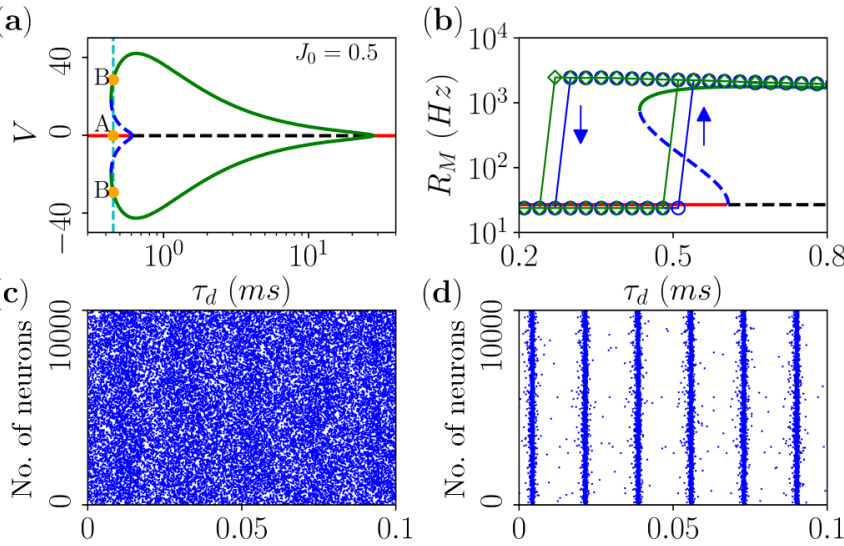

(d)
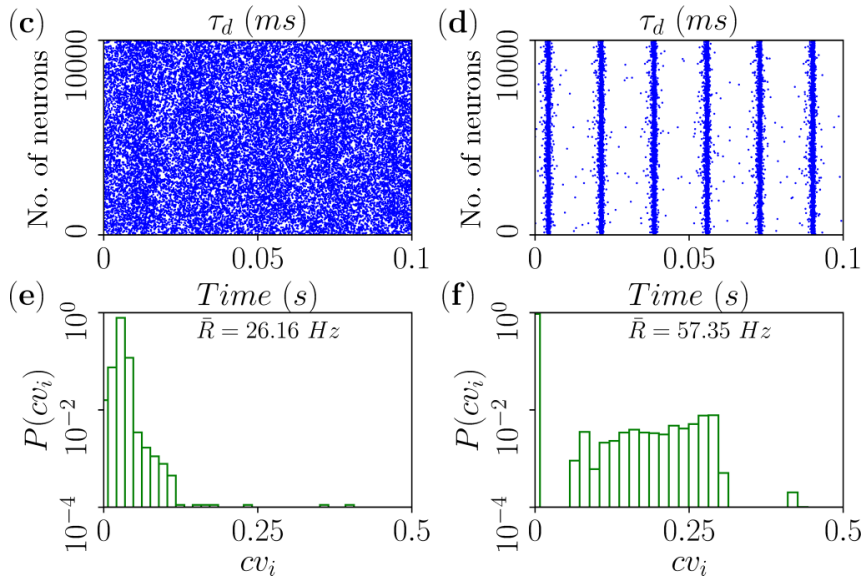

FIG. 5. High structural heterogeneity: subcritical Hopf bifurcation. (a) Bifurcation diagram of the MF model analogous to the one reported in Fig. 4(a). The supercritical (subcritical) Hopf bifurcation takes place at $\tau_{2}^{(H)}=27.96 \mathrm{~ms}\left(\tau_{1}^{(H)}=0.61 \mathrm{~ms}\right)$ and the saddle node of limit cycles is at $\tau^{(S)}=0.43 \mathrm{~ms}$. The capital letters in (a) denote two stationary states corresponding to the same synaptic timescales $\tau_{d}=0.45 \mathrm{~ms}$. This state is denoted in Fig. 2(b) by a yellow diamond. The network dynamics corresponding to these states is reported in (c) and (e) $A$ and (d) and (f) $B$. Also shown are (c) and (d) the raster plots and (e) and (f) the distribution of the $\left\{\mathrm{cv}_{i}\right\}$ of the single neurons. (b) Maximal values of the rate $R_{M}$ obtained by performing adiabatic simulations by first increasing and then decreasing the synaptic time $\tau_{d}$ (green diamonds) for $N=10000$ and (blue circles) for $N=20000$; the arrows denote the jump from one state to the other. The MF results are also displayed: red solid (black dashed) lines refer to stable (unstable) foci, while green solid (blue dashed) lines refer to stable (unstable) limit cycles. The parameters are the same as in Fig. 4 except for $J_{0}=0.5$; the parameters for the adiabatic simulations are $\Delta \tau_{d}=0.03 \mathrm{~ms}, \tau_{d}^{(0)}=0.21 \mathrm{~ms}$, and $\tau_{d}^{(1)}=0.81 \mathrm{~ms}$.

occurs at $\tau_{d}<\tau_{1}^{(H)}$ and the system returns from the oscillatory state to the asynchronous one at $\tau_{d}$, which is definitely smaller than $\tau^{(S)}$. These are finite-size (and possibly also finite-time) effects. Indeed, as shown in Fig. 5(b), by increasing $N$, the transition points approach the MF ones.

\section{B. Low structural heterogeneity}

We consider now a relatively low value of the structural heterogeneity, i.e., $\Delta_{0}=0.3$, which for instantaneous synapses can sustain a dynamically balanced state [21]. Let us first consider a relatively large coupling, namely, $J_{0}=17.0$; the corresponding bifurcation diagram for the MF model is reported in Fig. 6(a). This is quite similar to the one previously shown for high structural heterogeneity in Fig. 4(a). However, peculiar differences are observable at the level of network simulations. Indeed, in this case COs are present for all the $\tau_{d}$ values considered, even if these correspond to stable foci 

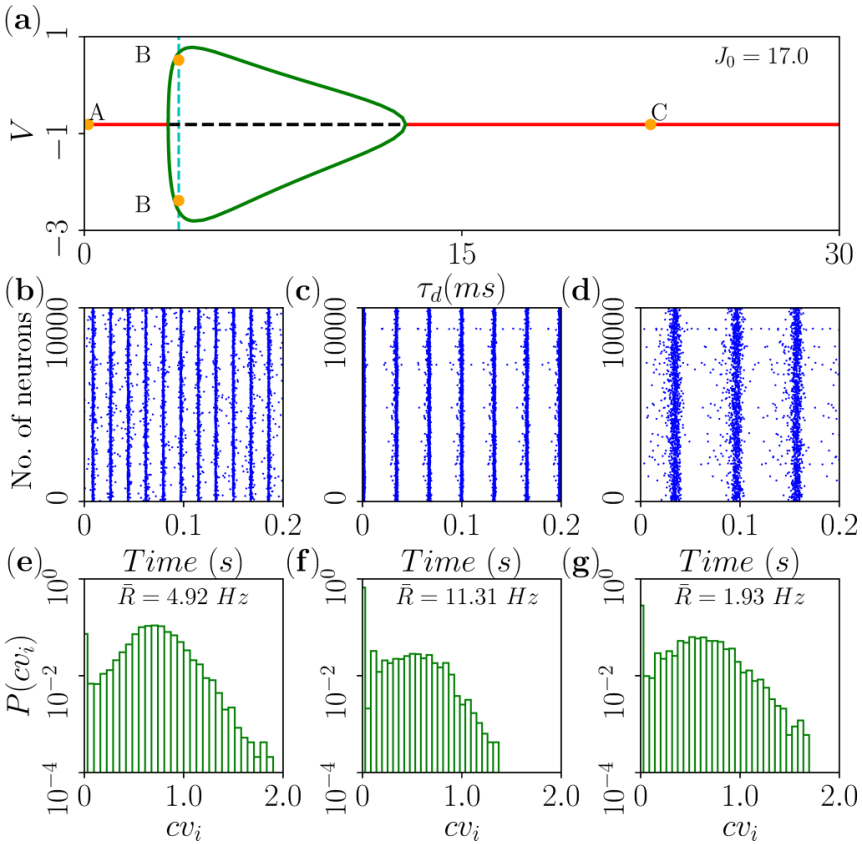

FIG. 6. Low structural heterogeneity: supercritical Hopf bifurcation. The panels displayed here are analogous to the ones in Fig. 4. In this case the supercritical Hopf bifurcations occur for $\tau_{1}^{(H)}=3.33 \mathrm{~ms}$ and $\tau_{2}^{(H)}=12.61 \mathrm{~ms}$. The stationary states in (a) corresponding to $A, B$, and $C$ refer to $\tau_{d}=0.15,3.75$, and $22.5 \mathrm{~ms}$, respectively. The parameters are the same as in Fig. 4 except for $\Delta_{0}=0.3$. and $J_{0}=17$. States $A, B$, and $C$ are denoted as in Figs. 2(a) and 3(c) by a red circle, a blue square, and a green triangle, respectively.

in the mean field [states $A$ and $C$ in Fig. 6(a)] as evident from the raster plots shown in Figs. 6(b) and 6(d). In particular, we measured the following frequencies for the observed COs: $v_{\mathrm{osc}} \simeq 57 \mathrm{~Hz}$ for state $A, v_{\mathrm{osc}} \simeq 30 \mathrm{~Hz}$ for $B$, and $v_{\mathrm{osc}} \simeq$ $16 \mathrm{~Hz}$ for $C$. Furthermore, the network dynamics is now definitely more irregular than for high $\Delta_{0}$ with distributions $P\left(\mathrm{cv}_{i}\right)$ centered around $\mathrm{cv}_{i}=1$ for the states $A$ and $C$ in Fig. 6(a) corresponding to stable foci in the MF formulation [see Figs. 6(e) and $6(\mathrm{~g})$ ] and with $P\left(\mathrm{cv}_{i}\right)$ extending towards values around $\mathrm{cv}_{i} \simeq 1$ for the oscillatory state $B$, as shown in Fig. 6(i). This irregularity in the spike emissions is a clear indication that now the dynamics is mostly fluctuation driven due to the dynamically balanced dynamics observable in the sparse network for sufficiently low structural heterogeneity. Furthermore, as shown in [21] for instantaneous synapses, these current fluctuations are able to turn the macroscopic damped oscillations towards the stable foci, observable in the MF model, in sustained COs in the network. The origin of the COs observable for the state $B$ is indeed different, since in this case sustained oscillations emerge due to a supercritical Hopf bifurcation both in the mean field and in the network dynamics.

By decreasing the synaptic coupling $J_{0}$ [Fig. 7(a)] we observe in the MF phase diagram the emergence of regions where the oscillations coexist with the stable focus in proximity to a subcritical Hopf bifurcation, analogously to what has been reported for high heterogeneity [see Fig. 5(a)]. At variance with that case, we have now in the network a
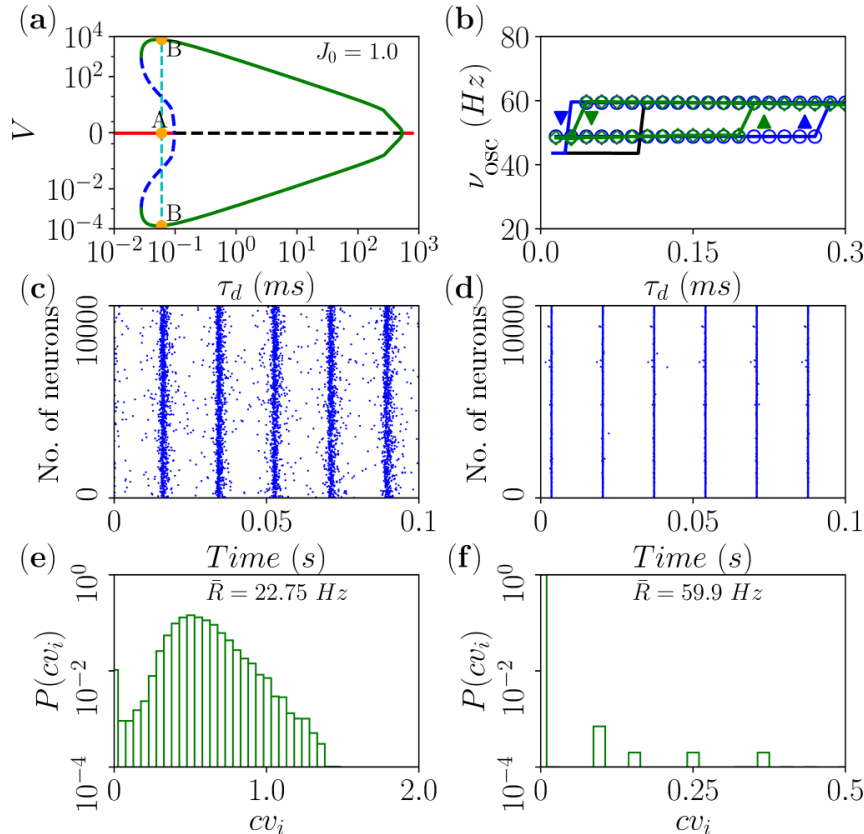

FIG. 7. Low structural heterogeneity: subcritical Hopf bifurcation. Except for (b), the panels are analogous to those in Fig. 5. In the present case the subcritical Hopf bifurcation occurs at $\tau_{1}^{(H)}=$ $0.097 \mathrm{~ms}$, while the supercritical Hopf bifurcation occurs at $\tau_{2}^{(H)}=$ $531.83 \mathrm{~ms}$ and the saddle node of limit cycles at $\tau^{(S)}=0.028 \mathrm{~ms}$; the coexisting states $A$ and $B$ shown in (a) refer to $\tau_{d}=0.06 \mathrm{~ms}$. This state is denoted in Figs. 2(a), 2(c), and 3(a) by a yellow diamond. (b) Frequency of collective oscillations as measured via adiabatic simulations for $N=2000$ by considering $T_{s}=90 \mathrm{~ms}$ (blue circles) and $T_{s}=1500 \mathrm{~ms}$ (green diamonds); the transient time $T_{t}=15 \mathrm{~ms}$ is unchanged. The solid lines in (b) refer to the MF results, namely, the black line to $v_{D}$ and the blue one to the limit cycle frequency $v_{O}$. The parameters are as in Fig. 6 except for $J=1.0$ and the adiabatic simulations are $\Delta \tau_{d}=0.015 \mathrm{~ms}, \tau_{d}^{(0)}=0.015 \mathrm{~ms}$, and $\tau_{d}^{(1)}=0.30 \mathrm{~ms}$.

bistability between two COs whose origin is different: One emerges via a Hopf bifurcation and it is displayed in Fig. 7(d), while the other is sustained by the irregular spiking associated with the balanced state and the corresponding raster plot is shown in Fig. 7(c). In particular, the latter COs are associated with large cv values [Fig. 7(e)] typical of a balanced regime, while the other COs are extremely regular as shown in Fig. 7(f) resembling the dynamics of a highly synchronized system.

In order to analyze the coexistence region, we report in Fig. 7(b) the frequencies $v_{\text {osc }}$ of the collective oscillations as measured via adiabatic simulations of the network (symbols). Furthermore, the MF results for $v_{D}$ associated with the foci and the frequencies $v_{O}$ of the limit cycles are also reported in the figure as black and blue solid lines, respectively. The frequencies of the COs in both states are reasonably well captured by the MF approach. Furthermore, the two frequencies can be quantitatively associated with fast and slow $\gamma$ oscillations. The comparison reveals that the COs induced by microscopic irregular firing exist far beyond $\tau_{1}^{(H)}$, despite that here the unique stable solution predicted by the mean field should be the almost synchronized bursting state shown 

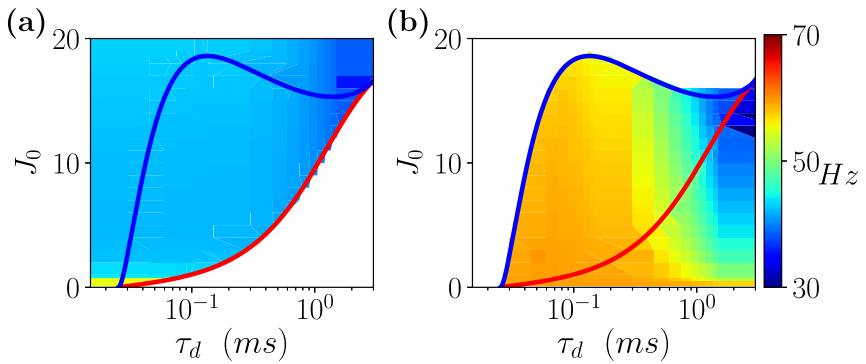

FIG. 8. Coexisting fast and slow $\gamma$ oscillations. (a) Frequencies $v_{D}$ associated with the damped oscillations towards the stable foci. (b) Frequencies $v_{O}$ of the limit cycles. Red lines refer to the subcritical Hopf boundaries, while the blue lines refer to saddle-node bifurcations of limit cycles. The other parameters are $I_{0}=0.25$ and $\Delta_{0}=0.3$.

in Fig. 7(d). On the other hand, the backward transition is almost coincident with the MF prediction for $\tau^{(S)}$ as displayed in Fig. 7(b). As reported in Fig. 7(b), we observe that also the forward transition value approaches $\tau_{1}^{(H)}$ by increasing the duration $T_{s}$ of the adiabatic steps. Therefore, this result suggests that the observed discrepancies are due to finitetime (and possibly finite-size) effects affecting the network simulations.

\section{COEXISTENCE OF SLOW AND FAST $\gamma$ OSCILLATIONS}

In the preceding section we showed, for a specific choice of the parameters, that fast and slow collective $\gamma$ oscillations can coexist. However, the phenomenon is observable in the whole range of coexistence of the stable foci and the stable limit cycles. In particular, in Fig. 8 we show in the $\left(\tau_{d}, J_{0}\right)$ plane the frequencies $v_{D}$ associated with the damped oscillations towards the MF focus [Fig. 8(a)] and the frequencies $v_{O}$ of the limit cycles [Fig. 8(b)]. It is evident that $v_{D} \simeq 30-40 \mathrm{~Hz}$, while the frequencies of the limit cycle $v_{O}$ are of the order of $60 \mathrm{~Hz}$; thus in the network we expect to observe coexisting COs characterized by slow and fast rhythms in a wide range of parameters.

For this parameter set $v_{D}$ seems to depend only slightly on $\tau_{d}$ and $J_{0}$. On the contrary, the frequency $v_{O}$, characterizing the more synchronized events, is influenced by these parameters. In particular, $v_{O}$ decreases for increasing IPSP time duration, similarly to what is observed experimentally for cholinergic induced $\gamma$ oscillations in the hippocampus in vitro [50]. Moreover, barbiturate, a drug often used as an anxiolytic, is known to increase IPSP time duration [73] and slow down $\gamma$ oscillations [74], in accordance with our scenario. Furthermore, for $\tau_{d}>1 \mathrm{~ms}$ the increase of $J_{0}$ leads to a decrease of $v_{D}$, similarly to the effect of alcohol that induces an increase of inhibition associated with a decrease in $\gamma$ oscillation frequencies measured in the human visual cortex [75].

The coexistence of fast and slow $\gamma$ COs is a quite general phenomenon not limited to the specific network topology we employed, i.e., that associated with the Lorentzian in-degree distribution. Indeed, as shown in Appendix A, it can be observed also for a sparse Erdős-Rényi network.

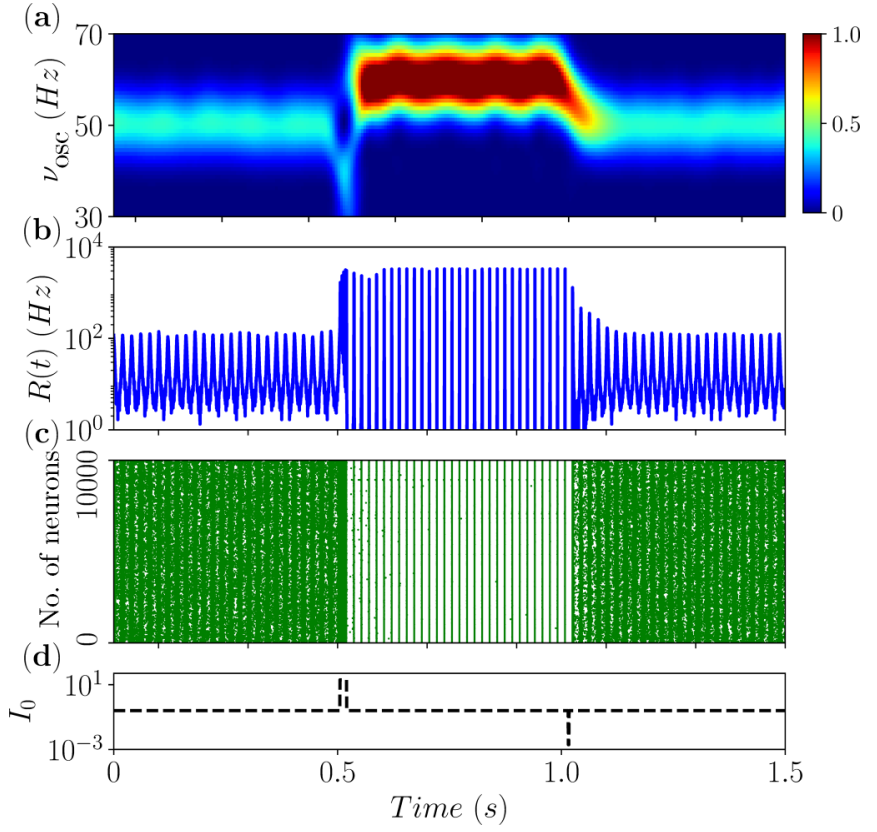

FIG. 9. Switching from fast (slow) to slow (fast) $\gamma$ oscillations. The results are from the switching experiments described in the text: (a) spectrogram of the mean membrane potential $V$, (b) the firing rate $R(t)$, (c) the raster plot, and (d) the stimulation protocol reporting the average external DC. The parameters are the same as in Fig. 7 (in particular $\tau_{d}=0.06 \mathrm{~ms}$ ) except for $T_{\mathrm{sh}}=0.015 \mathrm{~s}, T_{\mathrm{sl}}=0.0015 \mathrm{~s}$, $I_{1}=0.25, I_{2}=20.0, I_{3}=0.012$, and $\Delta I_{3}=0.01$.

\section{A. Switching $\gamma$ rhythms}

As an additional aspect we will consider the possibility to develop a simple protocol to drive the system from slow $\gamma$ COs to fast ones (and vice versa) in the bistable regime. Let us consider the case where the network is oscillating with slow $\gamma$ frequency as shown in Fig. 9 for $I_{0} \equiv I_{1}=0.25$. The protocol to drive the system in the fast $\gamma$ band consists in delivering a step current $I_{2}$ to all the neurons for a very limited time interval $T_{\mathrm{sh}}$. In this way the system is transiently driven in a regime where oscillatory dynamics is the only stable solution. As a matter of fact, the neurons remain in a high-frequency state even after the removal of the stimulation, when $I_{0}$ returns to the initial value $I_{1}$ (see Fig. 9). In order to desynchronize the neurons and to recover the slow $\gamma \mathrm{COs}$, we delivered random quenched DCs $I_{0}(i)$ (with $i=1, \ldots, N$ ) to the neurons for a time period $T_{\mathrm{sl}}$. The currents $I_{0}(i)$ are taken from a flat distribution with a very low average value $I_{3}$ and a width $\Delta I_{3}$ corresponding to a parameter range where the MF foci are the only stable solutions. As shown in Fig. 9, in this case, to drive the system from fast to slow $\gamma$ oscillations it was sufficient to apply the perturbation for a much smaller period $T_{\mathrm{sl}} \ll T_{\mathrm{sh}}$.

Let us now try to characterize in more detail the observed switching transitions. This can be done by considering the MF bifurcation diagram in terms of the external DC $I_{0}$ reported in Fig. 10(a) for the parameters examined. The diagram reveals a subcritical Hopf bifurcation taking place at $I^{(H)} \simeq 0.43$ and a region of bistability extending from $I^{(S)} \simeq 0.06$ to $I^{(H)}$. Therefore, if we consider a DC in the bistable interval (namely, 

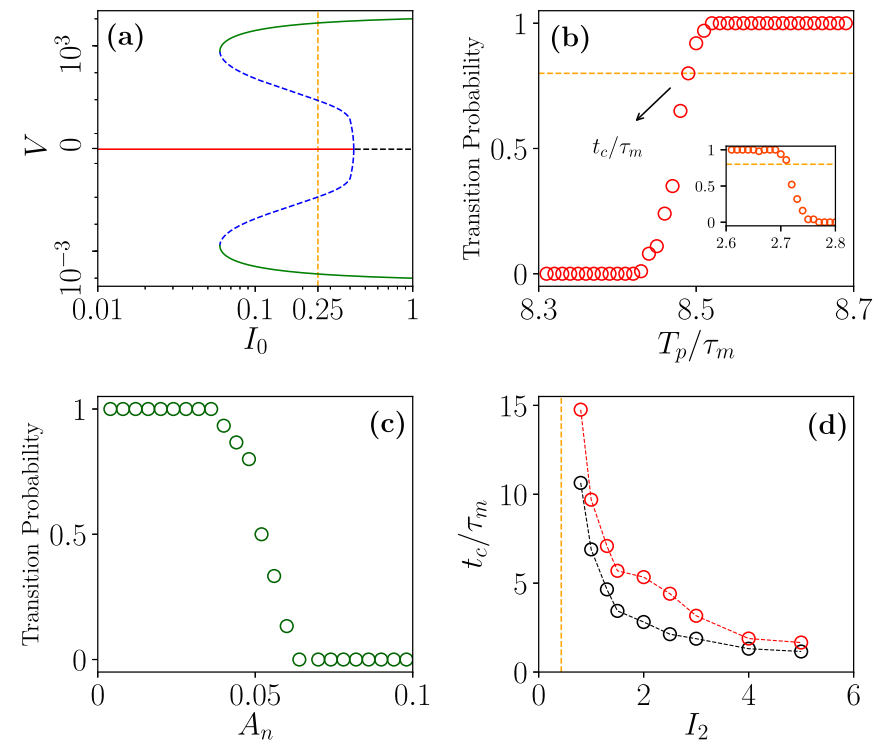

FIG. 10. Statistics of the switching transitions. (a) Bifurcation diagram of the MF model reporting the extrema of the mean membrane potential $V$ as a function of $I_{0}$ displaying stable (solid lines) and unstable solutions (dashed lines) for foci (red and black) and limit cycles (green and blue). The vertical dashed orange line refers to $I_{0}=0.25$. (b) Transition probability as a function of $T_{P}$. The orange dashed line denotes the $80 \%$ for $I_{2}=1.0$. The inset shows the data for the transition from fast to slow $\gamma$ for $I_{3}=0.03$; in both cases $A_{n}=0.05$. (c) Transition probability as a function of the noise amplitude $A_{n}$ for $I_{2}=1$ and $T_{P}=8.48 \tau_{m}$. (d) Crossing times $t_{c}$ versus the perturbation amplitude $I_{2}$ for noise levels $A_{n}=0.02$ (black circles) and 0.07 (red circles). The vertical orange line indicates the value $I^{(H)}$. (b)-(d) Transition from slow to fast $\gamma$ COs. The inset in (b) shows the transition from fast to slow $\gamma$. The parameters are the same as in Fig. 7.

$\left.I_{0} \equiv I_{1}=0.25\right)$ and we prepare the system in the slow $\gamma$ regime, a transition to the fast $\gamma$ COs will be observable whenever the DC is increased to a value $I_{0} \equiv I_{2}>I^{(H)}$. However, if we return to the bistable regime at $I_{0} \equiv I_{1}$, after delivering the perturbation $I_{2}$ for a time interval $T_{P}$, it is not evident in which regime (fast or slow) the system will end up. Thus we have measured the transition probability from slow to fast $\gamma$ for different $T_{P}$ and $I_{2}$ by following the protocol reported in Sec. II B. We analyzed these transitions in the presence of a small additive noise on the membrane potentials of amplitude $A_{n}$, somehow encompassing the many sources of noise present in neural circuits.

The results shown in Fig. 10(b) for $I_{2}=1.0$ and $A_{n}=0.05$ reveal that even if $I_{2}>I^{(H)}$ the perturbation should be applied for a minimal time interval $T_{P}>t_{c} \simeq 0.12 \mathrm{~s}$ to induce the transition to the fast $\gamma$ COs in at least the $80 \%$ of cases. It is interesting to note that the noise amplitude can play a critical role on the switching transition. Indeed, the increase of $A_{n}$ can desynchronize the fast $\gamma$ regime even for $T_{P}>t_{c}$ [see Fig. 10(c)]. Therefore, $t_{c}$ depends critically not only on $I_{2}$ but also on $A_{n}$ : As expected, by increasing $I_{2}$ the crossing time drops rapidly towards zero, while the switching transition is delayed to longer times for larger $A_{n}$ [see Fig. 10(d)].
Concerning the transition from fast to slow $\gamma$, this occurs in an irreversible manner only for an amplitude of the perturbation $I_{3}<I^{(S)}$; an example is shown in the inset of Fig. 10(b). Even though the switching transition can be observed also for $I_{3}>I^{(S)}$, this will be much more complex due to the competition of the two stable states in the interval $I_{0} \in$ $\left[I^{(S)}: I^{(H)}\right]$ and more specific protocols should be designed to obtain the desynchronization of the system.

\section{B. The $\theta-\gamma$ cross-frequency coupling}

So far we have described a simple protocol where external constant stimulations to the inhibitory network can drive the neural population from one state to the other. However, $\gamma$ oscillations are usually modulated by $\theta$ oscillations in the hippocampus and in the neocortex during movement and REM sleep [34,44]. This has recently inspired a series of optogenetic experiments in vitro intended to reproduce the effect of the $\theta$ forcing and the activity observed in vivo $[39,48,49]$. To draw a connection with these experiments, we decided to consider a periodic stimulation of all neurons in the network as

$$
I_{0}(t)=I_{\theta}\left[1-\cos \left(2 \pi v_{\theta} t\right)\right]
$$

where the phase of the $\theta$ forcing is defined as $\theta(t)=2 \pi v_{\theta} t$. The term appearing in (13) corresponds to the synaptic input received by the neurons; in order to compare this forcing term with the LFPs experimentally measured in $[35,46]$ which reveal $\theta$ oscillations, one should recall that the LFP corresponds to the electrical potential measured in the extracellular medium around neurons [76]. Therefore, for a meaningful comparison with the synaptic input (13) the sign of the LFP should be reversed. This is consistent with the observations reported in $[35,46]$ that the maximum of activity of the excitatory (pyramidal) cells is observed corresponding to the minimum of the LFP.

We considered the network dynamics in the presence of the periodic forcing (13) and additive noise on the membrane potentials (with zero mean and amplitude $A_{n}$ ). As shown in Fig. 11, the response of the system to the forcing is controlled by the value of the amplitude $I_{\theta}$ in (13): For small $I_{\theta} \leqslant 0.20$ one observes only slow $\gamma$ COs; for intermediate values of the amplitude $0.20<I_{\theta} \leqslant 0.32$ the slow and fast $\gamma$ COs coexist; for $I_{\theta} \geqslant 0.32$ only fast oscillations are present.

For small $I_{\theta}$, as one can appreciate from the raster plot in Fig. 12(c), the firings of the neurons are quite irregular and the bursts involve only a limited number of neurons, as confirmed also by the low values of $\rho$ during the firing activity [see Fig. 12(c)]. Furthermore, the corresponding spectrogram in Fig. 11(k) reveals that the power is concentrated at frequencies below $50 \mathrm{~Hz}$ and that the amplitude of the spectrum has a modulated structure as a function of the phase. This is confirmed by the analysis of the power of the spectrum $P S$ $(P L)$ restricted to the slow (fast) $\gamma$ band [see Fig. 11(n)]. These are indications of $\theta$-nested $\gamma$ oscillations, as confirmed by the instantaneous firing rate given in Fig. 11(1), which reveals also an evident PA coupling between the $\gamma$ phases and the $\theta$ forcing. In this case we considered a quite small value of the amplitude $A_{n}$ of the extrinsic noise, in order to compare our findings with in vitro experiments. 


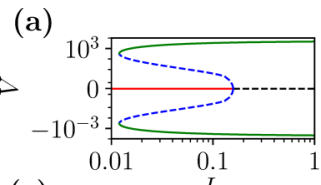

(b)
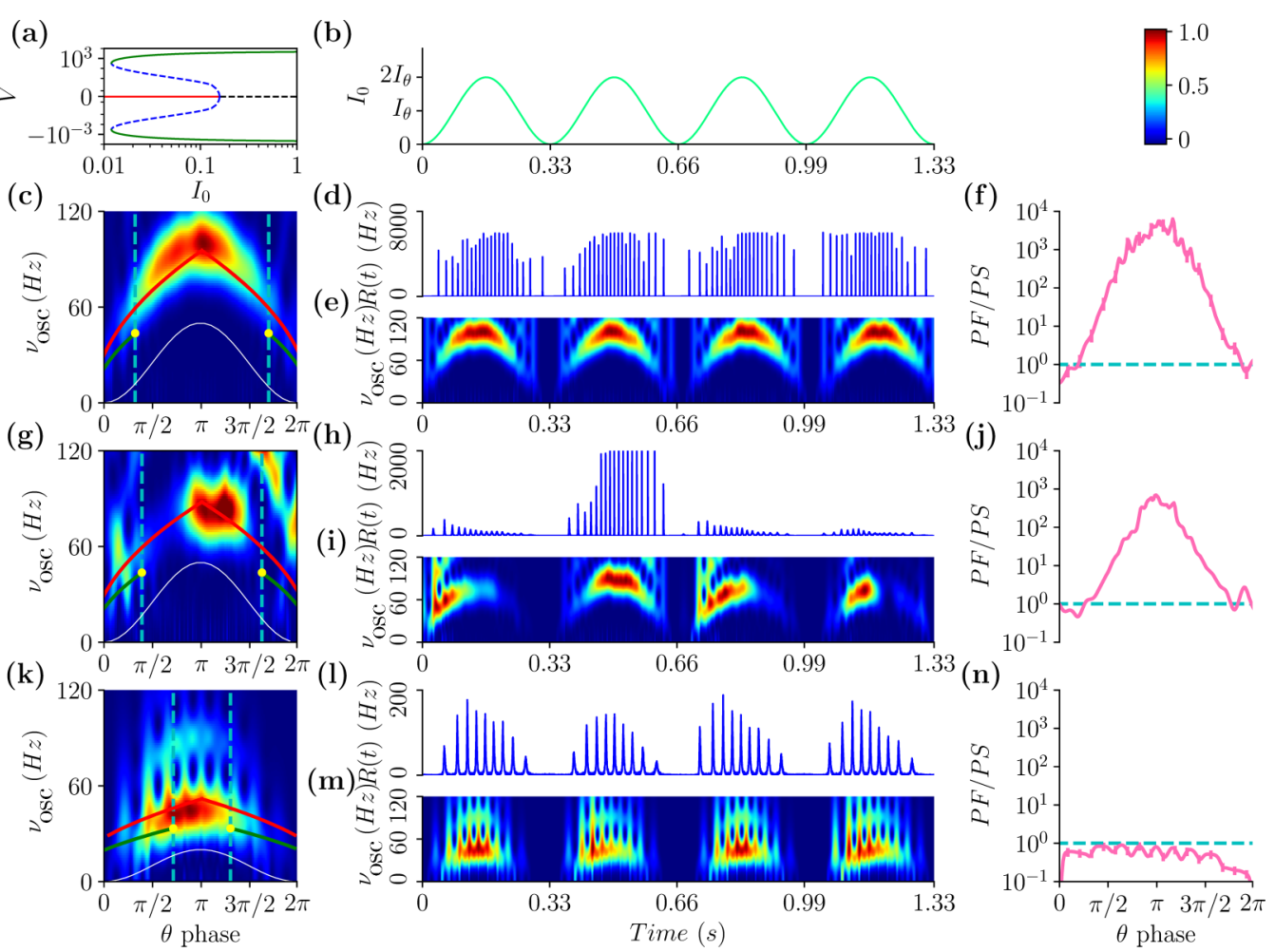

FIG. 11. Fast and slow $\gamma$ oscillations entrainment with the $\theta$ forcing. (a) Bifurcation diagram of the MF model analogous to the one shown in Fig. 10(a). (b) The $\theta$ forcing (13) versus time. Panels (c)-(f) refer to forcing with $I_{\theta}=0.35$ and noise amplitude $A_{n}=1$; (g)-(j) to $I_{\theta}=0.30$ and $A_{n}=1 ;(\mathrm{k})-(\mathrm{n})$ to $I_{\theta}=0.1$ and $A_{n}=1 \times 10^{-3}$. In the left column are reported averaged spectrograms as a function of the $\theta$ phase, together with $v_{D}$ (green solid line), $v_{O}$ (red solid lines) and the forcing in arbitrary units (white solid line). The vertical blue dashed lines indicate the stability boundaries for the focus solution; the focus is unstable for $\theta$ phases within such lines. The middle column displays an instance over four $\theta$ cycles of the corresponding spectrograms and of the instantaneous firing rates $R(t)$. The right column shows the ratio $P F / P S$ of the power contained in the fast $\left(50<v_{\mathrm{osc}}<100 \mathrm{~Hz}\right)$ and slow $\left(30 \leqslant v_{\mathrm{osc}} \leqslant 50 \mathrm{~Hz}\right) \gamma$ bands as a function of the $\theta$ phase. In this case the error bars are displayed, but are almost invisible on the reported scale. The parameters are $J_{0}=1, \tau_{d}=0.15 \mathrm{~ms}, \Delta=0.3$, and $K=1000$. For the simulations we considered $N=10000$ and $v_{\theta}=3 \mathrm{~Hz}$. The data for the spectrograms (left column) have been obtained by averaging over 30 $\theta$ cycles and those for $P F / P S$ (right column) over 400 cycles.

Indeed, these results resemble experimental observations of $\theta$-nested $\gamma$ oscillations induced in vitro by sinusoidal optical stimulation at $\theta$ frequency in the medial entorhinal cortex (mEC) [49] and in the CA1 [39] and CA3 [48] of the hippocampus. In all these experiments single neurons spiked quite irregularly, while the collective dynamics was oscillatory, analogously to our dynamics as shown in Figs. 11(1) and 12(c). As previously discussed, these COs are induced by intrinsic fluctuations and characterized at a MF level by frequencies $\simeq v_{D}$ (green solid line), which represents a reasonable estimation of the position of the maxima of the spectrogram as shown in Fig. 11(k).

The situation is quite different for sufficiently large forcing amplitude, where the neuronal dynamics becomes quite regular and highly synchronized, as evident from Figs. 11(d) and 12(a) and by the value of $\rho$ reported in Fig. 12(a). In this case the power is concentrated in the fast $\gamma$ band and it is maximal, corresponding to the largest value of $I_{0}$ occurring at $\theta=\pi$ [see Figs. 11(c) and 11(f)]. Furthermore, the profile of the maximal power in the spectrogram follows reasonably

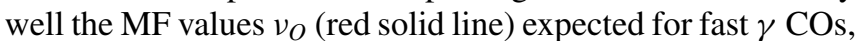
as evident from Fig. 11(c). For these large currents we have a sort of pathological synchronization usually observable in connection with neuronal diseases. In particular, highly synchronized fast $\gamma$ oscillations have been observed in patients with neocortical epilepsy [77].

The most interesting situation occurs for intermediate amplitudes; specifically, we considered $I_{\theta}=0.30$. As evident from Figs. 11(h) and 12(b), in this case the network dynamics can vary noticeably from one $\theta$ cycle to the next, due to the switching from one $\gamma$ regime to the other occurring erratically, induced by the larger extrinsic noise here employed (namely, $\left.A_{n}=1\right)$ to mimic in vivo conditions. This is particularly evident in Fig. 12(b), where the network dynamics reveals a large variability as testified by the level of synchronization from one cycle to another. However, by averaging over a sufficiently large number of cycles we can identify stationary features of this dynamics. In particular, we observe that the values of maximum power in the averaged spectrum correspond to different $\theta$ phases for the slow and fast $\gamma$ COs, namely, for slow $\gamma$ the maximal activity is observable at small angles, while for fast $\gamma$ this corresponds to the largest value of the forcing current (13) [see Figs. 11(g) and 11(j)]. Furthermore, on average we observe a steady increase of $\rho$ within a $\theta$ cycle 


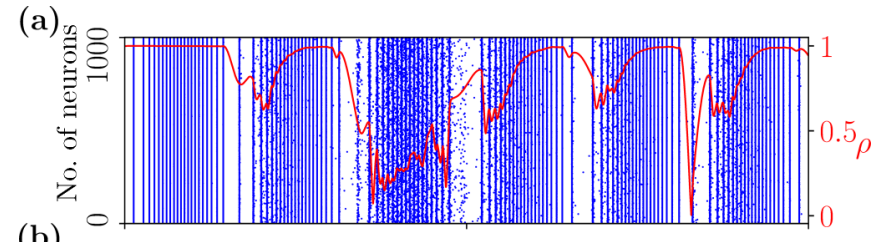

(b)

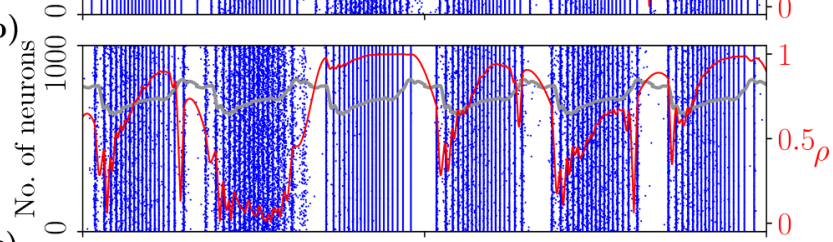

(c)

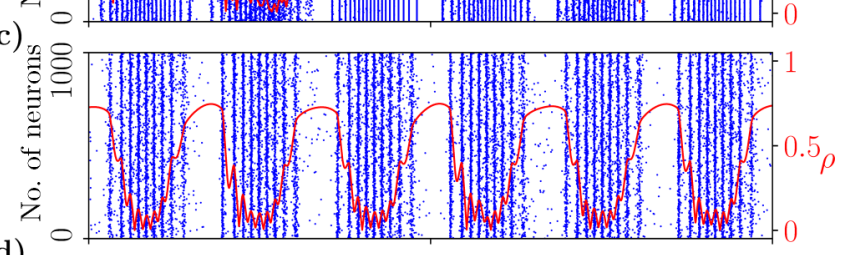

(d)

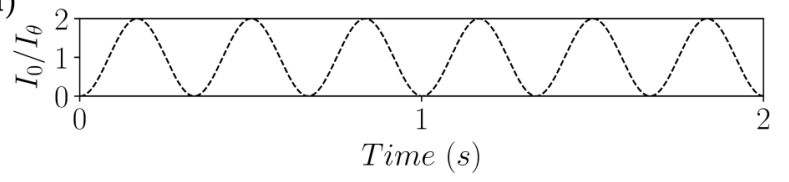

FIG. 12. Level of synchronization of fast and slow $\gamma$ oscillations. (a)-(c) Raster plots and Kuramoto order parameter $\rho$ (red solid curve) in the presence of $\theta$ forcing (13) for (a) $I_{\theta}=0.35$, (b) $I_{\theta}=$ 0.3 , and (c) $I_{\theta}=0.1$ with amplitude of noise as in Fig. 11. The gray curve in (b) represents the value of $\rho$ for one $\theta$ period averaged over $50 \theta$ cycles. (d) The $\theta$ forcing profile versus time. The parameters are $J_{0}=1, \tau_{d}=0.15 \mathrm{~ms}, \Delta=0.3, K=1000, N=10000$, and $v_{\theta}=3 \mathrm{~Hz}$.

[see the gray line in Fig. 12(b)], confirming that slow irregular $\gamma$ COs are mostly observable at small angles, while fast, more regular $\gamma$ COs set in at $\theta \geqslant \pi$.

These findings resemble the experimental results reported in [46] for the region CA1 of the hippocampus in freely moving rats, where it has been reported that slow $\gamma$ power was peaked around $\theta \simeq 0.4 \pi$ and fast $\gamma$ power around $\theta \simeq$ $\pi$, corresponding also to the maximum activity of excitatory place cells. Similar results have been reported in [35] for the slow $\gamma$ rhythm; however, in those experiments fast $\gamma$ (referred to as intermediate $\gamma$ ) occurs earlier in the $\theta$ cycle. Recent experiments obtained on behaving rats singled out a wide cycle-to-cycle variability of the frequency of CA1 $\theta$-nested $\gamma$ oscillations and an overlap of the frequency bands associated with slow and fast $\gamma$ oscillations [78]. These results do not contradict previous findings which were mainly based on spectral features obtained by averaging over many cycles $[35,46]$. Our results confirm the absence of this contradiction. Indeed, the instantaneous spectrograms reported in Fig. 11(i) show a large cycle-to-cycle variability, while the average spectrogram shown in Fig. 11(g) displays distinct peaks for slow and fast $\gamma$ COs at specific $\theta$ angles.

The network response to the external periodic forcing (13) can be interpreted in terms of an adiabatic variation of the external current whenever the timescale of the forcing term is definitely slower with respect to the neuronal timescales, i.e., $\tau_{m}$ and $\tau_{d}$. Since this is the case, we can try to understand the observed dynamics at a first level of approximation by employing the bifurcation diagram of the MF model obtained for a constant DC $I_{0}$, which is shown in Fig. 11(a) for the set of parameters considered here. The diagram reveals that the system bifurcates via a subcritical Hopf bifurcation from the asynchronous state to regular oscillatory behavior at a current $I^{(H)} \simeq 0.159$ and that the region of coexistence of stable foci and limit cycles is delimited by a saddle-node bifurcation occurring at $I^{(S)} \simeq 0.012$ and by $I^{(H)}$.

The forcing current (13) varies over a $\theta$ cycle from a value $I_{0}=0$ at $\theta=0$ up to a maximal value $I_{0}=2 I_{\theta}$ at $\theta=\pi$ and returns to zero at $\theta=2 \pi$. Since the forcing current will start from a zero value, we expect that the network will start oscillating with slow $\gamma$ frequencies associated with the stable focus, which is the only stable solution at small $I_{0}<I^{(S)}$. Furthermore, if $I_{\theta}<I^{(H)} / 2$ the system will remain always in the slow $\gamma$ regime during the whole forcing period, since the focus is stable up to the current $I^{(H)}$.

For amplitudes $I_{\theta}>I^{(H)} / 2$ we expect a transition from slow to fast COs for a $\theta$ phase $\theta^{(H)}=\arccos \left[\left(I_{\theta}-I^{(H)}\right) / I_{\theta}\right]$ corresponding to the crossing of the subcritical Hopf bifurcation. Since this transition is hysteretic the system will remain in the fast regime until the forcing current does not become extremely small, namely, $I_{0}<I^{(S)}$, corresponding to a $\theta$ phase $\theta^{(S)}=2 \pi-\arccos \left[\left(I_{\theta}-I^{(S)}\right) / I_{\theta}\right]$.

The analysis performed is quasistatic and does not take into account the time spent in each regime. If $I_{\theta} \gg I^{(H)}$ the time spent by the system in the slow $\gamma$ regime is extremely reduced, because $\theta^{(H)} \simeq 0$ and $\theta^{(S)} \simeq 2 \pi$, and this explains why for large $I_{\theta}$ we essentially observe only fast $\gamma$. On the other hand, we find only slow $\gamma$ COs for $I_{\theta}$ up to 0.20 , a value definitely larger than $I^{(H)} / 2$, and this due to the fact that a finite crossing time is needed to jump from one state to the other, as discussed in the preceding section.

Let us now focus on the case $I_{\theta}=0.3$, where we observe the coexistence of fast and slow $\gamma$ COs. As already mentioned, we have stable foci in the range $I_{0} \in\left[0: I^{(H)}\right]$; this in terms of $\theta$ angles obtained via the relationship (13) for $I_{\theta}=0.3$ corresponds to an interval $\theta / \pi \in[0: 0.34]$, roughly matching the region of the spectrogram reported in Fig. $11(\mathrm{~g})$ where the maximum power of slow $\gamma$ oscillations is observable. Due to the hysteretic nature of the subcritical Hopf transition, even if the forcing current $I_{0}(\theta)$ decreases for $\theta \rightarrow 2 \pi$, we would not observe slow $\gamma$ at large $\theta$ angles. This is indeed confirmed by the average value of the order parameter $\rho$ [gray solid line in Fig. 12(b)], which reveals an almost monotonic increase with $\theta$ over a cycle.

For currents $I_{0}>I^{(H)}$ only the limit cycles (corresponding to fast $\gamma$ COs with frequencies $v_{O}$ ) are stable. Indeed, the maximum of the power spectrum for fast $\gamma$ COs occurs for $\theta \simeq \pi$ where $I_{0} \simeq 0.6>I^{(H)}$ is maximal.

As a last point, let us examine if the coexistence of fast and slow $\gamma \mathrm{COs}$ is related to some form of PP locking between $\theta$ forcing and $\gamma$ oscillations [35,40]. As is evident from Figs. 13(a) and 13(b), the $\theta$ forcing at $v_{\theta}=10 \mathrm{~Hz}$ locks the collective network dynamics, characterized by the mean membrane potential and by the $\gamma$ phase defined in Sec. II C. In particular, for this specific time window we observe for each $\theta$ oscillation exactly six $\gamma$ oscillations of variable duration, slower at the extrema of the $\theta$ window and faster in the central 

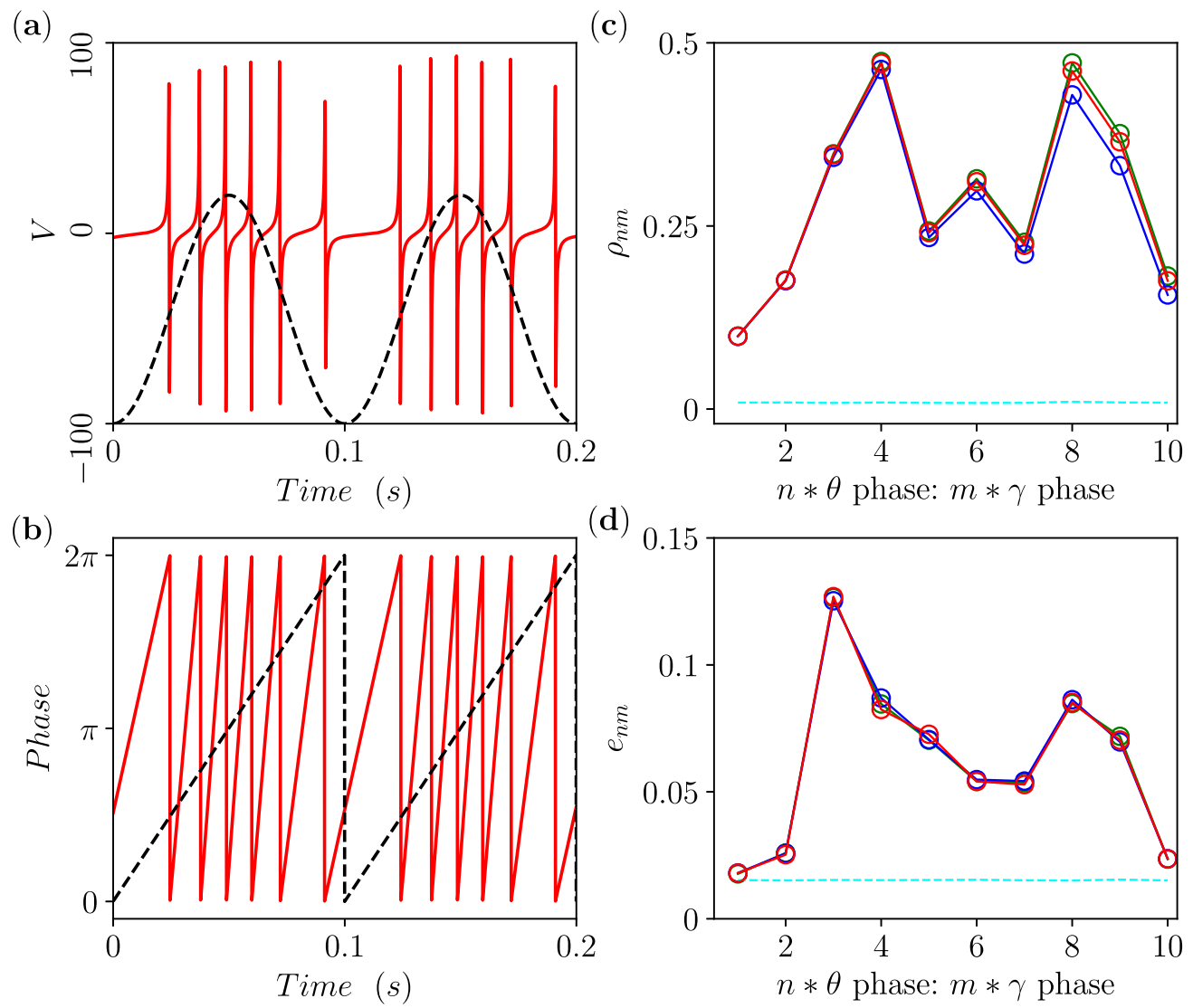

FIG. 13. Phase-phase coupling $n: m$ between $\theta$ forcing and $\gamma$ oscillations (a) and (b) Locking of the $\gamma$ oscillations to the external $\theta$ forcing: (a) average membrane potential $V$ versus time, with the black dashed line showing the forcing (13) in arbitrary units, and (b) $\gamma$ (red solid line) and $\theta$ (black dashed line) phases for the corresponding time interval. (c) Kuramoto order parameter $\rho_{n m}$ for the phase difference $\Delta_{n m}(t)$ for time windows of duration $T_{W}=0.1 \mathrm{~s}$ (black), $0.5 \mathrm{~s}$ (red), and $1 \mathrm{~s} \mathrm{(blue)} \mathrm{averaged} \mathrm{over} 70<M<700$ different realizations. (d) Normalized entropy $e_{n m}$ for a time window $T_{W}=0.5 \mathrm{~s}$ averaged over $M=140$ realizations (black). The surrogate data are also reported corresponding to random permutation (red) and a time shift (blue) of the original data averaged over $M=100$ independent realizations. The reported data refer to the simulation of the spiking network subject to the external forcing (13) with additive noise on the membrane potentials. The parameters are the same as in Fig. 11 except for $I_{\theta}=0.3, v_{\theta}=10 \mathrm{~Hz}$, and $A_{n}=1.0 \times 10^{-3}$. The histogram of $\Delta_{n m}(t)$ employed for the estimation of $e_{n m}$ has been evaluated over $M=50$ bins. The results refer only to phases associated with $\gamma$ frequencies in the band 30-100 Hz. The error bars in (c) and (d) are of the order of the size of the symbols. The cyan dashed lines show the significance levels $(\mathrm{c}) \rho^{(S)}=0.009$ and $(\mathrm{d}) e^{(S)}=0.016$.

part, in agreement with the expected coexistence of $\gamma$ rhythms of different frequencies.

Let us quantify these qualitative observations by considering statistical indicators measuring the level of $n: m$ synchronization for irregular/noisy data over a large number of $\theta$ cycles. In particular, we will employ the Kuramoto order parameter $\rho_{n m}$ and the normalized entropy $e_{n m}$ introduced in Sec. IIC measured over time windows of duration $T_{W}$ and averaged over many different realizations.

As shown in Figs. 13(c) and 13(d), both these indicators exhibit two maxima showing the existence of two different lockings between $\theta$ and $\gamma$ oscillations for $n: m$ equal to $\simeq 3-4$ and $\simeq 8$, thus corresponding to slow and fast $\gamma\left(v_{\theta}=10 \mathrm{~Hz}\right)$. Following [61], in order to test if the reported PP couplings are significant, we have estimated $\rho_{n m}$ over time windows of increasing duration, namely, from 0.1 to $1 \mathrm{~s}$. As shown in Fig. 13(c), the measured values do not vary substantially even by increasing $T_{W}$ by a factor 10 . This is a clear indication of the stationarity of the PP locking phenomenon analyzed here [61]. Furthermore, we measured $e_{n m}$ also for surrogate data obtained by random permutation and by a time shift (for the exact definitions see Sec. II C and [61]); the values obtained for these surrogate data are almost indistinguishable from the original ones [see Fig. 13(d)]. These results suggest that more effective approaches able to distinguish a true locked state from spurious locking should be developed.

Finally, the significance level of the reported measurements has been evaluated by randomly shuffling the time stamps of the $\gamma$ phases, denoted by $\rho^{(S)}$ and $e^{(S)}$ [dashed lines in Figs. 13(c) and 13(d)]. The values of $\rho^{(S)}$ and $e^{(S)}$ are definitely smaller than those of the corresponding indicators corresponding to the observed PP lockings, thus confirming their significance.

\section{CONCLUSION}

In this paper we have shown in terms of an effective mean field that in a sparse balanced inhibitory network with finite synaptic decay COs can emerge via supercritical or subcritical Hopf bifurcations from a stable focus. Furthermore, in the 
network (for sufficiently low structural heterogeneity) the macroscopic focus turns out to be unstable towards microscopic fluctuations in the firing activity leading to the emergence of COs characterized by a frequency corresponding to that of the damped oscillations towards the MF focus. Therefore, in proximity to the subcritical Hopf bifurcations, the coexistence of two COs with different origins is observable: slow (fast) $\gamma$ oscillations being fluctuation (mean) driven.

From our analysis it emerges that two ingredients are needed to observe coexisting slow and fast $\gamma$ COs: the sparseness in the connections and the dynamical balance of the network activity. In particular, the sparseness has a twofold effect at the macroscopic level and at the microscopic level. In a mean-field framework the randomness in the in-degree distribution can be reinterpreted as a quenched disorder in the synaptic couplings, which gives rise to the coexistence of stable foci and limit cycles. However, in a fully coupled network with heterogeneous parameters we would not observe strong irregular fluctuations at the level of single neurons, analogous to Poissonian-like firings usually observed in the cortex $[56,57,79]$. These can emerge only in sparsely connected networks $[19,20]$. Moreover, the balance mechanism guarantees that the irregular spiking dynamics will not disappear in the thermodynamic limit [21,23-25]. These persistent microscopic fluctuations are able to trigger slow $\gamma$ COs in the network, which coexist with fast $\gamma$ COs corresponding to the limit cycle solutions in the mean field. These two ingredients usually characterize real brain networks, where our prediction that slow (fast) $\gamma$ oscillations are associated with more (less) irregular neuronal dynamics can be experimentally tested, e.g., by measuring the coefficient of variation associated with these two states. Furthermore, previous theoretical analysis of $\gamma$ oscillations based on two interacting Wilson-Cowan rate models with different synaptic times revealed only the possible coexistence of two stable limit cycles, both corresponding to tonic collective firing, i.e., mean-driven COs [54].

A fundamental improvement of the effective mean field introduced here should include in the formulation also the current fluctuations due to the sparseness. A possible strategy to incorporate these intrinsic noise sources in an exact meanfield formulation, going beyond the Ott-Antonsen ansatz [8], could rely on the circulant cumulant expansion recently applied to an ensemble of oscillators [80] and an ensemble of QIF neurons [81] in the presence of extrinsic noise.

Furthermore, a standard approach to obtain a macroscopic description of the neuronal network dynamics is based on derivation of the Fokker-Planck equation for the distribution of the membrane potentials. This formalism has been fully developed for sparse networks of leaky integrate-and-fire neurons in $[19,20]$. A preliminary analysis based on this approach for the QIF network examined here and in [21] suggests that fluctuation-driven COs emerge due to an instability of the asynchronous state [82].

Our model is not meant to explicitly replicate the dynamics of specific brain areas but rather to illustrate fundamental mechanisms by which slow and fast $\gamma$ oscillations may arise and coexist due to local network inhibitory features. However, several phenomena we reported resemble experimental results obtained for different brain regions in vitro as well as in vivo. Our findings though can stimulate further experiments or lead to alternative interpretations of the existing data.

Of particular interest is the possibility, analyzed in Sec. V A, to switch from a $\gamma$ rhythm to another by performing transient stimulations. This mechanism can allow a single inhibitory population to pass from a coding task to another following an external sensory stimulus. Indeed, it has been shown that distinct $\gamma$ rhythms are involved in different coding processes, namely, fast $\gamma$ in new memory encoding, while slow $\gamma$ has been hypothesized to promote memory retrieval [83].

On the one hand, pathological synchronization is usually associated with neuronal diseases $[15,84,85]$. Aberrant $\gamma$ oscillations have been observed in several cognitive disorders, including Alzheimer's disease, fragile X syndrome, and neocortical epilepsy $[77,83]$. Furthermore, deep brain stimulation (DBS) techniques have been developed along the years to treat some of these diseases, e.g., essential tremor and Parkinson's disease [86-88]. We have presented a simple model exhibiting the coexistence of highly synchronized states and asynchronous or partially synchronized regimes. Therefore, our model can represent a simple benchmark to test new DBS protocols to obtain eventually less invasive technique to desynchronize pathological states [89-91] or to restore healthy $\gamma$ rhythms, as suggested in [83].

Moreover, the richness of the dynamical scenario present in this simple model indicates possible future directions where intrinsic mechanisms present in real neural networks such as spike frequency adaptation could permit a dynamical alternation between different states. In this direction, a slow variable like adaptation could drive the system from "healthy" asynchronous or oscillatory dynamics to periods of pathological extremely synchronous regimes, somehow similar to epileptic seizure dynamics [92].

In Sec. VB we analyzed the emergence of COs in our network in the presence of an external $\theta$ forcing, in order to draw a connection with recent experimental investigations devoted to analyzing the emergence of $\gamma$ oscillations in several brain areas in vitro under sinusoidally modulated $\theta$-frequency optogenetic stimulations [39,48,49]. For low forcing amplitudes, our network model displays $\theta$-nested $\gamma$ COs at frequencies $\simeq 50 \mathrm{~Hz}$ joined with irregular spiking dynamics. These results are analogous to the ones reported for the CA1 and CA 3 of the hippocampus in $[39,48]$. Moreover, $\theta$-nested oscillations with similar features have been reported also for the mEC [49], but for higher $\gamma$ frequencies.

Furthermore, for intermediate forcing amplitudes we observe the coexistence of slow and fast $\gamma$ oscillations, which lock to different phases of the $\theta$ rhythm, analogously to what has been reported for the rat hippocampus during exploration and REM sleep [35,46]. The $\theta$-phase preferences displayed in our model by the different $\gamma$ rhythms are due to the hysteretic nature of the subcritical Hopf bifurcation crossed during the $\theta$ stimulation. Finally, for sufficiently strong forcing, the model is driven in the fast $\gamma$ regime.

Our analysis suggests that a single inhibitory population can generate locally different $\gamma$ rhythms and lock to one or the other in the presence of a $\theta$ forcing. In particular, we have shown that fast $\gamma$ oscillations are locked to a strong excitatory input, while slow $\gamma$ COs emerge when excitation 
and inhibition balance. These results can be useful in revealing the mechanism behind slow and fast $\gamma$ oscillations reported in several brain areas, namely, the hippocampus [45], olfactory bulb [93], ventral striatum [94], visual cortical areas [95], and neocortex [44]. Particularly interesting is the clear evidence reported in [44] that different $\gamma$ rhythms, phase locked to the hippocampal $\theta$ rhythm, can be locally generated in the neocortex. Therefore, future studies could focus on this brain region to test for the validity of the mechanisms here reported.

The CA1 of the hippocampus is where most of the experimental studies on $\theta-\gamma$ oscillations have been performed. Despite the experimental evidence that different $\gamma$ oscillations emerging in the CA1 at different $\theta$ phases are a reflection of synaptic inputs originating from the CA3 and mEC [46,47], this does not exclude the possibility that a single CA1 inhibitory population can give rise to different $\gamma$ rhythms depending on the network state [45]. This hypothesis is supported by experimental evidence showing that a large part of CA1 interneurons in vivo can lock to both slow and fast $\gamma[35,46,47]$ and that in vitro $\gamma$ rhythms can be locally generated in various regions of the hippocampus due to optogenetic stimulations $[39,48,49]$ or pharmalogical manipulation [50-53]. However, much work remains to be done to clarify if local mechanisms can give rise to coexisting $\gamma$ rhythms also in the CA1.

Another interesting aspect, revealed quite recently, concerns the wide cycle-to-cycle variability in the $\theta$-nested $\gamma$ oscillations observed during spatial exploration and memoryguided behavior in CA1 [78] and in dentate gyrus [96]. In particular, in these papers the authors show not only that different $\gamma$ bands can be excited during different $\theta$ cycles but also that the spectral range of these bands overlap. This is consistent with our analysis for intermediate forcing amplitude reported in Figs. 11 and 12, where we show that the dynamics from one cycle to the next one is extremely variable and that the frequency bands associated with slow and fast COs slightly overlap around $40 \mathrm{~Hz}$.

At variance with previous results for purely inhibitory populations reporting noise sustained $\mathrm{COs}$ in the range 100-200 Hz [16], our model displays slow $\gamma$ rhythms characterized by irregular firing of the single neurons. Therefore, in our case it is not necessary to add an excitatory population to the inhibitory one to slow down the rhythm and to obtain oscillations in the $\gamma$ range as done in [30,97]. Evidence has been recently reported pointing out that $\gamma$ oscillations can emerge locally in the CA1 induced by the application of kainate due to purely inhibitory mechanisms [53]. However, other studies point out that in the same area of the hippocampus excitatory and inhibitory neurons should interact to give rise to oscillations in the $\gamma$ range [39,52]. Preliminary results obtained for QIF networks with a sinusoidal $\theta$ forcing show that $\theta$-nested $\gamma$ oscillations with similar features can emerge for purely inhibitory networks as well as for mixed excitatoryinhibitory networks [98].

As shown in Sec. III B, the same kind of bifurcation diagram can be observed by considering the external excitatory drive as well as the self-disinhibition of the recurrently coupled inhibitory population. This suggests that in our model the same scenarios reported in Sec. V for an excitatory $\theta$ forcing can be obtained by considering an external inhibitory population which transmits rhythmically its activity to the target population. This somehow mimics the pacemaker $\theta$ activity of a part of the medial septum interneurons on the interneurons of the hippocampus experimentally observed in [99]. This subject should be addressed in future studies due to its relevance in clarifying the origin of $\theta-\gamma$ oscillations in the hippocampus; however, it goes beyond the scopes of the present analysis.

In this paper we considered a model including the minimal ingredients necessary to reproduce the phenomenon of coexisting $\gamma$ oscillations corresponding to quite simple (namely, periodic) collective regimes. However, the introduction of synaptic delay in the model can lead to more complex coexisting states, like quasiperiodic and even chaotic solutions, as recently shown for fully coupled networks in $[63,100]$. The inclusion of delay in our model can enrich the dynamical scenario, maybe allowing the mimicking of further aspects of the complex patterns of activity observed in the brain, e.g., sharp-wave ripples observed in the hippocampus which are fundamental for memory consolidation [101]. Due to the large variety of interneurons present in the brain and in particular in the hippocampus [102], a further step in rendering our model more realistic would consist in considering multiple inhibitory populations characterized by different neuronal parameters. By manipulating the influence of a population on the others it would be interesting to investigate the possible mechanisms to switch COs from one $\gamma$ rhythm to another, following the richness of the bifurcation scenarios presented in Figs. 2 and 3 .

The generality of the phenomena here reported is addressed in Appendixes A and B. In particular, in Appendix A we show that the mechanisms leading to the coexistence scenario of fast and slow $\gamma$ oscillations are not peculiar of Lorentzian in-degree distributions (which we employed to allow a comparison of the network simulations with the MF results) but that they are observable also in the more studied Erdős-Rényi sparse networks. Appendix B is devoted to the analysis of a suitable normal form which reproduces the dynamics of the mean field in proximity to the subcritical Hopf bifurcation. In particular, the noisy dynamics of the normal form reveals coexisting oscillations of different frequencies. More specifically, the addition of noise leads from damped oscillations towards the stable focus to sustained oscillations characterized by the same frequency. The latter result links our findings to the more general context of noise-induced oscillations for nonexcitable systems examined in various fields of research, namely, single-cell oscillations [103], epidemics [104], predator-prey interactions [105], and laser dynamics [106]. At variance with all previous studies, we have analyzed noise-induced oscillations coexisting and interacting with oscillations emerging from the Hopf bifurcation. Furthermore, the mechanism leading to the irregular fluctuations in our case is quite peculiar. Single-cell oscillations are believed to be driven by molecular noise, induced by the small number of molecules present in each cell, and therefore disappearing in the thermodynamic limit [107]. Recently, another possible mechanism leading to fluctuation amplification in a feedforward chain has been suggested as a pacemaking mechanism for biological systems; in this context the amplitude of the oscillations grows with the system size [108]. Instead, in 
our case, the dynamical balance provides intrinsic noise and oscillations of constant amplitude, essentially independent from the number of synaptic inputs (in-degree) and from the number of neurons in the network.

\section{ACKNOWLEDGMENTS}

We acknowledge useful discussions with D. Avitabile, D. Angulo-Garcia, D. Battaglia, F. Devalle, S. Keeley, E. Montbrió, S. Olmi, A. Politi, J. Rinzel, and R. Schmidt. The authors received partial financial support by the French Governement via the Excellence Initiative I-Site Paris Seine (Grant No. ANR-16-IDEX-008), the Labex MME-DII (Grant No. ANR11-LBX-0023-01), and the ANR Project ERMUNDY (Grant No. 18-CE37-0014-03).

\section{APPENDIX A: SLOW AND FAST $\gamma$ OSCILLATIONS IN ERDÖS-RÉNYI NETWORK}

In order to compare the network simulations with the MF results, we have considered in this article a Lorentzian distribution for the in-degrees. It is therefore important to show that the same phenomenology is observable by considering a more standard distribution, like the Erdős-Rényi (ER) one. The results of adiabatic simulations, shown in Fig. 14, confirm that also for ER networks a bistable regime, characterized by COs with different $\gamma$ - frequencies [see Fig. 14(b)], is indeed observable. In particular, slow $\gamma$ COs characterized by an average firing rate $\bar{R} \simeq 25 \mathrm{~Hz}$ and irregular neuronal
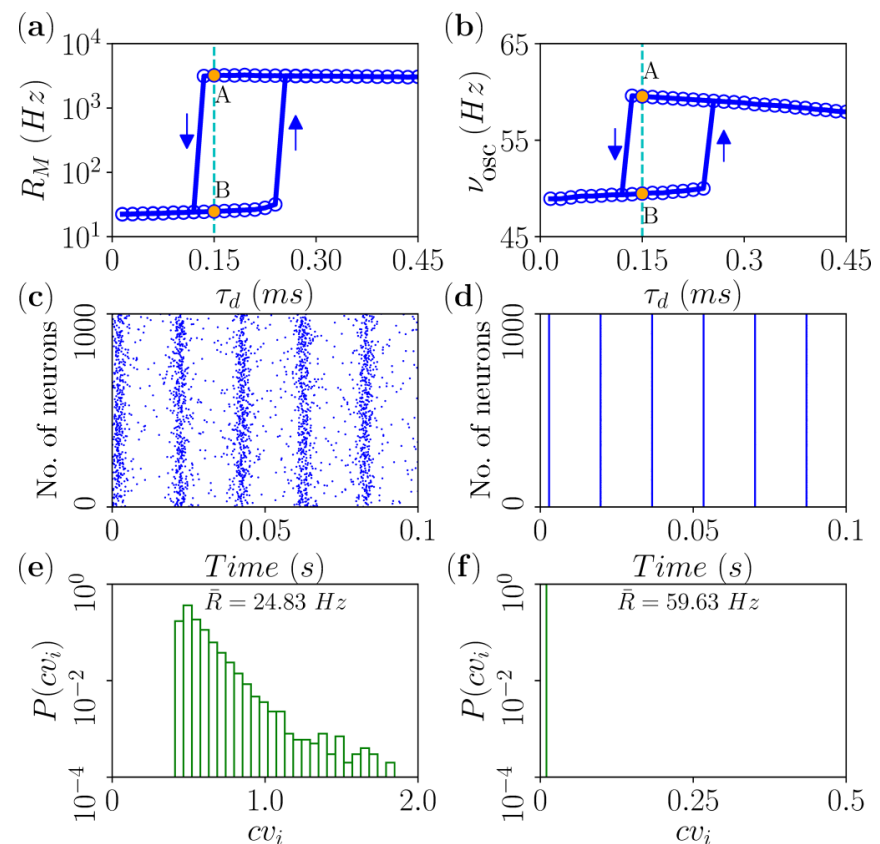

FIG. 14. Erdős-Rényi network. The results of adiabatic simulations for an ER network were obtained by varying the synaptic time $\tau_{d}$ : (a) maximal firing rates $R_{M}$ and (b) frequencies $v_{\text {osc }}$ of the COs. Two coexisting states $A$ and $B$ are considered at $\tau_{d}=0.15 \mathrm{~ms}$. (c) and (d) Raster plots and (e) and (f) distributions of $\mathrm{cv}_{i}$ are shown for states $A$ and $B$, respectively. The parameters for the simulations are $N=10000, K=1000, I_{0}=0.25, J_{0}=1.0, \Delta \tau_{d}=0.015 \mathrm{~ms}$, $\tau_{d}^{(0)}=0.015 \mathrm{~ms}$, and $\tau_{d}^{(1)}=0.45 \mathrm{~ms}$. firings [as shown in Figs. 14(c) and 14(e)] coexist with almost synchronized fast $\gamma$ COs with neurons tonically firing with $\bar{R} \simeq 60 \mathrm{~Hz}$ [see Figs. 14(d) and 14(f)].

\section{APPENDIX B: GENERAL MECHANISM FOR THE EMERGENCE OF COEXISTING OSCILLATIONS}

We investigate here the generality of the mechanism for the coexistence of COs observed in the network of QIF neurons. In particular, we show that this phenomenon occurs when in the MF model we have a focus coexisting with a limit cycle, while in the sparse network we have fluctuations sustained by the dynamical balance. If this is the mechanism, we expect to see a similar phenomenon whenever we consider a system in proximity to a subcritical Hopf bifurcation and we add noise of constant amplitude to the dynamics.

Therefore, to assess the generality of the phenomenon, we consider the normal form of a Hopf bifurcation in two dimensions leading to the birth of a limit cycle from an equilibrium, namely [109,110],

$$
\begin{aligned}
\tau_{m} \dot{x} & =\beta x-y+\sigma x r^{2}-(x+\gamma y) r^{4}+I_{1}, \\
\tau_{m} \dot{y} & =x+\beta y+\sigma y r^{2}+(\gamma x-y) r^{4}+I_{2},
\end{aligned}
$$

where $r^{2}=x^{2}+y^{2}, \tau_{m}=4 \mathrm{~ms}$ is an arbitrary timescale, $I_{1}(t)$ and $I_{2}(t)$ are generic external time-dependent forcing, $\beta$ is the bifurcation parameter, the parameter $\sigma$ sets the nature of the bifurcation, and $\gamma$ controls the frequency of the stable and unstable limit cycles. Notice that we added a quintic term, absent in the original normal form [109,110], in order to keep the values of $x$ and $y$ bounded while keeping the same bifurcation structure. For $I_{1}=I_{2}=0$ we will have a subcritical (supercritical) Hopf bifurcation for $\sigma=+1(\sigma=$ -1 ). In this case it is convenient to rewrite (B2) in polar
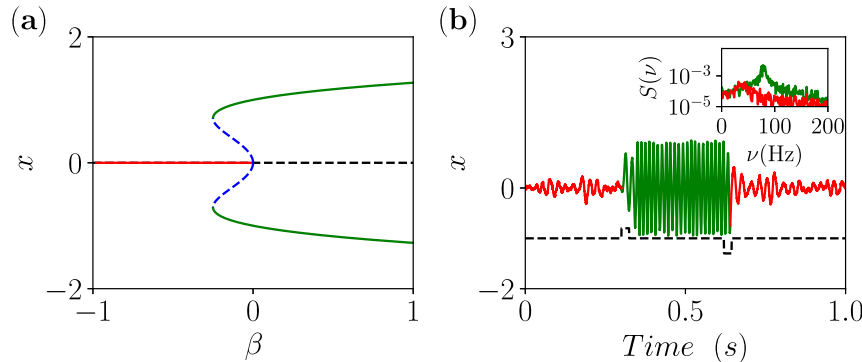

FIG. 15. (a) Bifurcation diagram for the variable $x$ as a function of the parameter $\beta$. Green (blue) lines indicate a stable (unstable) limit cycle and the red (black) line indicates a stable (unstable) focus. (b) Time trace of $x(t)$ in the presence of a zero-mean Gaussian noise of amplitude $A_{1}=A_{2}=0.14$, shown for fixed $\beta$ in the bistability region. An external pulse of current is added to the evolution equation for $x$ in (B2) for a time window of $56 \mathrm{~ms}$ to induce a switching between the oscillatory states (the black dashed line, shifted on the $x$ axis to be visible while the actual baseline value is zero). In the inset we show the power spectrum of the two different oscillatory regimes obtained over long time traces (hundreds of seconds) in order to check that the oscillations persist in time. The parameters are $\beta=-0.16, \sigma=1$, and $\gamma=1.5$. 
coordinates $(x, y)=(r \cos \phi, r \sin \phi)$, as follows:

$$
\begin{gathered}
\tau_{m} \dot{r}=\beta r+\sigma r^{3}-r^{5}, \\
\tau_{m} \dot{\phi}=1+\gamma r^{4} .
\end{gathered}
$$

The stationary solutions are $r=0$ corresponding to a stable focus characterized by relaxation oscillations with a frequency $v_{D} \simeq 39 \mathrm{~Hz}$ and stable and unstable limit cycles of amplitudes $r^{2}=\left(\sigma \pm \sqrt{\sigma^{2}+4 \beta}\right) / 2$.

In Fig. 15(a) we show the bifurcation diagram for $\sigma=$ +1 and $I_{1}=I_{2}=0$. We observe that the subcritical Hopf bifurcation occurs at $\beta=\beta_{c}=0$ and for $\beta<0$ it exists in a region where stable (green dots) and unstable (blue dashed line) limit cycles coexist with a stable focus (red line), exactly as it happens for the QIF MF model [see Fig. 7(a)]. The stable and unstable limit cycles merge at a saddle-node bifurcation located at $\beta=-\sigma^{2} / 4$.

As previously stated, the MF model cannot capture the endogenous fluctuations, naturally present in sparse balanced networks. In order to emulate this effect we consider $I_{1}(t)$ and $I_{2}(t)$ to be two independent and identically distributed Gaussian white-noise processes, i.e., $I_{q}(t)=A_{q} \xi_{q}(t)$ with $q=$ 1,2 , where $\xi_{q}(t)$ are random Gaussian distributed variables of zero average and unitary variance. In the presence of these additive noise terms and in proximity to the Hopf bifurcation, we observe the coexistence of two oscillatory regimes as shown in Fig. 15(b). One oscillation, characterized by higher amplitude (green line), corresponds to the limit cycle present in the non-noisy dynamics [green line in the bifurcation diagram shown in Fig. 15(b)]. The other oscillation is the result of a constructive role of noise that excites the stable focus, thus generating robust oscillations at the frequency $v_{D}$ (red line). Analogously to what was shown for the network of QIF neurons (see Fig. 8), it is possible to switch between the two kind of oscillations via a pulse current of positive (negative) amplitude with respect to the baseline [see the dashed line in Fig. 15(b)]. Moreover, the frequencies of the two oscillations, generated by two different mechanisms, correspond to slow and fast $\gamma$ oscillations as observable in the corresponding power spectra $S(v)$ shown in the inset of Fig. 15(b).
[1] A. Pikovsky and M. Rosenblum, Chaos 25, 097616 (2015).

[2] A. T. Winfree, The Geometry of Biological Time, 2nd ed., Interdisciplinary Applied Mathematics Vol. 12 (Springer, New York, 2001).

[3] Y. Kuramoto, Chemical Oscillations, Waves, and Turbulence (Springer Science + Business Media, 2012), Vol. 19.

[4] H. Hong, H. Chaté, H. Park, and L.-H. Tang, Phys. Rev. Lett. 99, 184101 (2007).

[5] J. D. Crawford, J. Stat. Phys. 74, 1047 (1994).

[6] S. H. Strogatz, Physica D 143, 1 (2000).

[7] J. Barre and D. Métivier, Phys. Rev. Lett. 117, 214102 (2016).

[8] E. Ott and T. M. Antonsen, Chaos 18, 037113 (2008).

[9] E. Montbrió, D. Pazó, and A. Roxin, Phys. Rev. X 5, 021028 (2015).

[10] G. Buzsaki, Rhythms of the Brain (Oxford University Press, Oxford, 2006).

[11] P. Fries, J. H. Reynolds, A. E. Rorie, and R. Desimone, Science 291, 1560 (2001).

[12] A. Bragin, G. Jandó, Z. Nádasdy, J. Hetke, K. Wise, and G. Buzsáki, J. Neurosci. 15, 47 (1995).

[13] J. Fell, P. Klaver, K. Lehnertz, T. Grunwald, C. Schaller, C. E. Elger, and G. Fernández, Nat. Neurosci. 4, 1259 (2001).

[14] A. K. Engel and W. Singer, Trends Cognit. Sci. 5, 16 (2001).

[15] W. Truccolo, O. J. Ahmed, M. T. Harrison, E. N. Eskandar, G. R. Cosgrove, J. R. Madsen, A. S. Blum, N. S. Potter, L. R. Hochberg, and S. S. Cash, J. Neurosci. 34, 9927 (2014).

[16] G. Buzsáki and X.-J. Wang, Annu. Rev. Neurosci. 35, 203 (2012).

[17] M. Bartos, I. Vida, and P. Jonas, Nat. Rev. Neurosci. 8, 45 (2007).

[18] N. Kopell and G. B. Ermentrout, in Handbook of Dynamical Systems, edited by B. Fiedler (Elsevier, Amsterdam, 2002), Vol. 2, p. 3.

[19] N. Brunel and V. Hakim, Neural Comput. 11, 1621 (1999).

[20] N. Brunel, J. Comput. Neurosci. 8, 183 (2000).
[21] M. di Volo and A. Torcini, Phys. Rev. Lett. 121, 128301 (2018).

[22] I. Vida, M. Bartos, and P. Jonas, Neuron 49, 107 (2006).

[23] C. van Vreeswijk and H. Sompolinsky, Science 274, 1724 (1996).

[24] A. Renart, J. de la Rocha, P. Bartho, L. Hollender, N. Parga, A. Reyes, and K. D. Harris, Science 327, 587 (2010).

[25] A. Litwin-Kumar and B. Doiron, Nat. Neurosci. 15, 1498 (2012).

[26] R. Rosenbaum and B. Doiron, Phys. Rev. X 4, 021039 (2014).

[27] J. Kadmon and H. Sompolinsky, Phys. Rev. X 5, 041030 (2015).

[28] R. W. Berg, A. Alaburda, and J. Hounsgaard, Science 315, 390 (2007).

[29] J. Barral and A. D. Reyes, Nat. Neurosci. 19, 1690 (2016).

[30] N. Brunel and X.-J. Wang, J. Neurophysiol. 90, 415 (2003).

[31] M. Monteforte and F. Wolf, Phys. Rev. Lett. 105, 268104 (2010).

[32] S. Jahnke, R.-M. Memmesheimer, and M. Timme, Phys. Rev. Lett. 100, 048102 (2008).

[33] M. Monteforte and F. Wolf, Phys. Rev. X 2, 041007 (2012).

[34] G. Buzsáki, Neuron 33, 325 (2002).

[35] M. A. Belluscio, K. Mizuseki, R. Schmidt, R. Kempter, and G. Buzsáki, J. Neurosci. 32, 423 (2012).

[36] J. Jacobs, Philos. Trans. R. Soc. B 369, 20130304 (2014).

[37] H. Zhang and J. Jacobs, J. Neurosci. 35, 12477 (2015).

[38] J. A. White, M. I. Banks, R. A. Pearce, and N. J. Kopell, Proc. Natl. Acad. Sci. USA 97, 8128 (2000).

[39] J. L. Butler, P. R. Mendonça, H. P. Robinson, and O. Paulsen, J. Neurosci. 36, 4155 (2016).

[40] P. Tass, M. G. Rosenblum, J. Weule, J. Kurths, A. Pikovsky, J. Volkmann, A. Schnitzler, and H.-J. Freund, Phys. Rev. Lett. 81, 3291 (1998).

[41] C. Zheng, K. W. Bieri, S. G. Trettel, and L. L. Colgin, Hippocampus 25, 924 (2015). 
[42] K. W. Bieri, K. N. Bobbitt, and L. L. Colgin, Neuron 82, 670 (2014).

[43] C. Zheng, K. W. Bieri, Y.-T. Hsiao, and L. L. Colgin, Neuron 89, 398 (2016).

[44] A. Sirota, S. Montgomery, S. Fujisawa, Y. Isomura, M. Zugaro, and G. Buzsáki, Neuron 60, 683 (2008).

[45] L. L. Colgin, Nat. Rev. Neurosci. 17, 239 (2016).

[46] L. L. Colgin, T. Denninger, M. Fyhn, T. Hafting, T. Bonnevie, O. Jensen, M.-B. Moser, and E. I. Moser, Nature (London) 462, 353 (2009).

[47] E. W. Schomburg, A. Fernández-Ruiz, K. Mizuseki, A. Berényi, C. A. Anastassiou, C. Koch, and G. Buzsáki, Neuron 84, 470 (2014).

[48] T. Akam, I. Oren, L. Mantoan, E. Ferenczi, and D. M. Kullmann, Nat. Neurosci. 15, 763 (2012).

[49] H. Pastoll, L. Solanka, M. C. van Rossum, and M. F. Nolan, Neuron 77, 141 (2013).

[50] A. Fisahn, F. G. Pike, E. H. Buhl, and O. Paulsen, Nature (London) 394, 186 (1998).

[51] R. D. Traub, M. O. Cunningham, T. Gloveli, F. E. LeBeau, A. Bibbig, E. Buhl, and M. Whittington, Proc. Natl. Acad. Sci. USA 100, 11047 (2003).

[52] A. N. Pietersen, P. D. Ward, N. Hagger-Vaughan, J. Wiggins, J. G. Jefferys, and M. Vreugdenhil, J. Physiol. 592, 605 (2014).

[53] M. T. Craig and C. J. McBain, J. Neurosci. 35, 3616 (2015).

[54] S. Keeley, A. A. Fenton, and J. Rinzel, J. Neurophysiol. 117, 950 (2016).

[55] A. Renart, R. Moreno-Bote, X.-J. Wang, and N. Parga, Neural Comput. 19, 1 (2007).

[56] D. Angulo-Garcia, S. Luccioli, S. Olmi, and A. Torcini, New J. Phys. 19, 053011 (2017).

[57] F. Devalle, A. Roxin, and E. Montbrió, PLoS Comput. Biol. 13, e1005881 (2017).

[58] S. Coombes and Á. Byrne, in Nonlinear Dynamics in Computational Neuroscience (Springer, Berlin, 2019), pp. 1-16.

[59] G. B. Ermentrout and N. Kopell, SIAM J. Appl. Math. 46, 233 (1986).

[60] J. A. Acebrón, L. L. Bonilla, C. J. Pérez Vicente, F. Ritort, and R. Spigler, Rev. Mod. Phys. 77, 137 (2005).

[61] R. Scheffer-Teixeira and A. B. Tort, eLife 5, e20515 (2016).

[62] S. Olmi, A. Politi, and A. Torcini, Europhys. Lett. 92, 60007 (2011).

[63] D. Pazó and E. Montbrió, Phys. Rev. Lett. 116, 238101 (2016).

[64] B. Ermentrout, Scholarpedia 2, 1399 (2007).

[65] G. Sperk, S. Furtinger, C. Schwarzer, and S. Pirker, Recent Advances in Epilepsy Research (Springer, Berlin, 2004), pp. 92-103.

[66] M. I. González, H. L. Grabenstatter, C. A. Cea-Del Rio, Y. C. Del Angel, J. Carlsen, R. P. Laoprasert, A. M. White, M. M. Huntsman, and A. Brooks-Kayal, Neurobiol. Dis. 77, 246 (2015).

[67] R. Dickinson, S. Awaiz, M. Whittington, W. Lieb, and N. Franks, Neuropharmacology 44, 864 (2003).

[68] D. A. McCormick and D. A. Prince, Proc. Natl. Acad. Sci. USA 82, 6344 (1985).

[69] C. J. Frazier, A. V. Buhler, J. L. Weiner, and T. V. Dunwiddie, J. Neurosci. 18, 8228 (1998).
[70] R. Pyle and R. Rosenbaum, Phys. Rev. E 93, 040302(R) (2016).

[71] I. D. Landau, R. Egger, V. J. Dercksen, M. Oberlaender, and H. Sompolinsky, Neuron 92, 1106 (2016).

[72] S. Olmi, A. Politi, and A. Torcini, J. Math. Neurosci. 2, 12 (2012).

[73] R. Nicoll, J. Eccles, T. Oshima, and F. Rubia, Nature (London) 258, 625 (1975).

[74] R. D. Traub, N. Spruston, I. Soltesz, A. Konnerth, M. A. Whittington, and J. G. Jefferys, Prog. Neurobiol. 55, 563 (1998).

[75] A. E. Campbell, P. Sumner, K. D. Singh, and S. D. Muthukumaraswamy, Neuropsychopharmacology 39, 2104 (2014).

[76] P. L. Nunez and R. Srinivasan, Electric Fields of the Brain: The Neurophysics of EEG, 2nd ed. (Oxford University Press, New York, 2006).

[77] G. A. Worrell, L. Parish, S. D. Cranstoun, R. Jonas, G. Baltuch, and B. Litt, Brain 127, 1496 (2004).

[78] V. Lopes-dos-Santos, G. M. van de Ven, A. Morley, S. Trouche, N. Campo-Urriza, and D. Dupret, Neuron 100, 940 (2018).

[79] E. Ullner and A. Politi, Phys. Rev. X 6, 011015 (2016).

[80] I. V. Tyulkina, D. S. Goldobin, L. S. Klimenko, and A. Pikovsky, Phys. Rev. Lett. 120, 264101 (2018).

[81] D. S. Goldobin and A. V. Dolmatova, Phys. Rev. Research 1, 033139 (2019).

[82] M. Segneri, M. di Volo, D. S. Goldobin, A. Politi, and A. Torcini (unpublished).

[83] A. J. Mably and L. L. Colgin, Curr. Opin. Neurobiol. 52, 182 (2018).

[84] C. Hammond, H. Bergman, and P. Brown, Trends Neurosci. 30, 357 (2007).

[85] A. Oswal, P. Brown, and V. Litvak, Curr. Opin. Neurol. 26, 662 (2013).

[86] J. S. Perlmutter and J. W. Mink, Annu. Rev. Neurosci. 29, 229 (2006).

[87] S. Breit, J. B. Schulz, and A.-L. Benabid, Cell Tissue Res. 318, 275 (2004).

[88] C. De Hemptinne, N. C. Swann, J. L. Ostrem, E. S. RyapolovaWebb, M. San Luciano, N. B. Galifianakis, and P. A. Starr, Nat. Neurosci. 18, 779 (2015).

[89] P. A. Tass, Biol. Cybern. 87, 102 (2002).

[90] O. V. Popovych, C. Hauptmann, and P. A. Tass, Phys. Rev. Lett. 94, 164102 (2005).

[91] R. J. Andrews, Ann. N.Y. Acad. Sci. 1199, 204 (2010).

[92] V. K. Jirsa, W. C. Stacey, P. P. Quilichini, A. I. Ivanov, and C. Bernard, Brain 137, 2210 (2014).

[93] L. M. Kay, J. Integr. Neurosci. 2, 31 (2003).

[94] M. A. A. van der Meer and A. D. Redish, Front. Integr. Neurosci. 3, 1 (2009).

[95] A. M. Bastos, J. Vezoli, C. A. Bosman, J.-M. Schoffelen, R. Oostenveld, J. R. Dowdall, P. De Weerd, H. Kennedy, and P. Fries, Neuron 85, 390 (2015).

[96] V. Douchamps, S. Ferte, D. Battaglia, and R. Goutagny (unpublished).

[97] C. Geisler, N. Brunel, and X.-J. Wang, J. Neurophysiol. 94, 4344 (2005). 
[98] M. Segneri, H. Bi, S. Olmi, and A. Torcini (unpublished).

[99] B. Hangya, Z. Borhegyi, N. Szilágyi, T. F. Freund, and V. Varga, J. Neurosci. 29, 8094 (2009).

[100] F. Devalle, E. Montbrió, and D. Pazó, Phys. Rev. E 98, 042214 (2018).

[101] G. Buzsáki, Hippocampus 25, 1073 (2015).

[102] G. Maccaferri and J.-C. Lacaille, Trends Neurosci. 26, 564 (2003).

[103] P. Thomas, A. V. Straube, J. Timmer, C. Fleck, and R. Grima, J. Theor. Biol. 335, 222 (2013).

[104] R. Kuske, L. F. Gordillo, and P. Greenwood, J. Theor. Biol. 245, 459 (2007).

[105] A. Rozenfeld, C. Tessone, E. Albano, and H. Wio, Phys. Lett. A 280, 45 (2001).
[106] O. V. Ushakov, H.-J. Wünsche, F. Henneberger, I. A. Khovanov, L. Schimansky-Geier, and M. Zaks, Phys. Rev. Lett. 95, 123903 (2005).

[107] J. M. Vilar, H. Y. Kueh, N. Barkai, and S. Leibler, Proc. Natl. Acad. Sci. USA 99, 5988 (2002).

[108] D. Fanelli, F. Ginelli, R. Livi, N. Zagli, and C. Zankoc, Phys. Rev. E 96, 062313 (2017).

[109] A. A. Andronov, E. A. Leontovich, I. I. Gordon, and A. G. Maier, Theory of Bifurcations of Dynamic Systems on a Plane (Israel Program for Scientific Translations, Jerusalem, 1971), Vol. 554.

[110] Y. A. Kuznetsov, Elements of Applied Bifurcation Theory, Applied Mathematical Sciences Vol. 112 (Springer Science + Business Media, New York, 2013). 